\title{
INVOCATION DU PARADIS PERDU \\ LES CHANTS DE BAMBARAS \\ DANS "SEGOU, MURAILLES DE TERRE"
}

\author{
Par \\ Amgad El-Zarif Atta Hassan* \\ amgadelzarif71@gmail.com
}

\section{Résumé}

Les chants Bambaras constituent un document linguistique (rythmique et significatif de la grande saga de Ségou à la fin du XVIIIème Siècle et au début du XIXème Siècle); ils ont manifesté un pan-bambara très significatif dans la sémiotique narrative du roman Ségou, Murailles de Terre: ils sont devenus une incarnation et une illustration vivantes proche d'un film d'animation en faisant un rappel audio-visuel de tous les éléments du Royaume de Ségou. Ce répertoire oral abandonné se fonctionne avant tout comme un déclencheur d'affichage des étapes de la splendeur et de la déchéance de l'empire bambara. Il n'est pas donc surprenant que la narration massive commence par un chant et se termine aussi par un chant. Cet article retrace le paradigme de ces chants, avec leurs sons, leurs rythmes et leurs valeurs sémantiques et stylistiques, qui pénètrent le tissu narratif pour créer des saillies symboliques particulières. Il suggère comment les limites de ces chants formant un corpus oral homogène qui a remanipulé les enjeux de la restauration compensée du pouvoir bambara dans l'espace de l'Afrique de l'Ouest.

Mots-clés: Oralité, chants, rythme, image, restauration

* Professeur adjoint en linguistique Française et chef du département de français- Faculté des Lettres - Al-Arich. 
«L'art est le seul langage qui se partage à la surface de la Terre sans distinction de nationalité ni de race, ces deux fléaux qui interdisent la communication entre les hommes. » (Maryse Condé, 2003, pp.195-196)

\section{INTRODUCTION}

Maryse Condé, dans la grande saga de Ségou: Ségou, Les Murailles de Terre (SMT) (1984) et Ségou, La Terre en miettes, (STM) (1985) planifie la subversion des mémoires de l'oralité [qui dépassent le] métissage culturel, la naissance du mélange littéraire." (Cisse, 2006, 213) Condé "habite [sa] langue, la langue de Maryse Condé. Une langue qui s'appelle français, mais qui est la [sienne]. [Elle] écri[t] dans une langue à [elle]." (Poinsot et Treiber, 2013, 6)

De la sorte, l'identité orthographique italique des Chants Bambaras ${ }^{1}$ (Ch.B) suspendus au milieu du texte romanesque de SMT les fait saillir comme un corps/corpus dont l'accrochage affirme l'hypothèse qu'ils se réfèrent à un héritage remis en exergue par l'épopée de Ségou. Toute leur "irrégularité langagière [...] était reçue comme langage populaire [...] liée au cotexte et au contexte sociolinguistique. " (Rouayrenc, 2015, 2)

Selon Senghor, (Cf., Ahmadou Singare, 2012) le chant en Afrique dispose de deux traits caractéristiques fondamentaux: la diversité des rythmes et l'étrangeté des images. De ce point de vue, nous tenterons dans cette étude, de scruter la possibilité que ces deux éléments constitutifs puissent exécuter l'invocation de la mémoire

(Invocation du paradis perdu...) $\quad$ Dr. Amgad El-Zarif 
de Ségou, un paradis perdu. Les $\mathrm{Ch}$. B, rappelant le poids de la tradition orale, ont pris une courbe liée à la ligne narrative, et aux états psychologiques des héros bambara (enthousiasme, joie, isolement, mort, amour, faux espoir, etc.)

Ceci nous amène à poser les deux questions suivantes: Est-ce qu'un certain nombre de chants folkloriques, populaires, oraux peut cristalliser une invocation audio-visuelle d'une civilisation éteinte? Quels sont les moyens rythmiques, sémantiques, stylistiques qui puissent dessiner les sentiments et les impressions des héros à travers ces chants? Afin de répondre à ces questions, nous avons déterminé la préférence comme corpus de l'étude le roman Ségou, Murailles de terre de Maryse Condé. Celle-ci a pu y transmettre (réécrire) parfaitement cet héritage oral au sein du tissu fictif en vue de réinterpréter les émotions /sentiments des héros vis-àvis de l'effondrement de la civilisation de Ségou.

Les Ch. B s'emparent de formes rythmiques, phonétiques, syntaxiques, sémantiques et énonciatives, etc... Ces aspects langagiers sont réorganisés pour la réincarnation de la gloire déchue de la culture de Ségou. Ceux-ci "présentent un brassage ethnique important." (Ebongue, 2013,161) et un écart d'un système linguistique exclu du lecteur occidental en se référant aux particularités du parler de Ségou.

Les Ch. B construisent également "édifice de croyances, d'idées, d'habitudes mentales intériorisées 
constituant comme la mythologie du groupe." (Zumthor, 2008, 170) Sous une forme historique et dynamique, SMT cède un "modèle hypotextuel oral " (Granel, 1993, 661) à travers une opération textuelle compensatoire; celle de la poésie orale, "une parole spontanée." (Boula de Mareuil, $r .1 \leqslant, 9)$ Les Ch. B construisent un discours homogène issu uniquement de la reprise du quotidien de Ségou.

Les subtilités du langage des Ch.B s'avanceraient à un rythme et à un sens de l'image foudroyante à son écart poétique et son pigment de réminiscence du passé en tant d' "ethnotextes figés " et " [de] formes dynamiques. " (Martin-Granel, 1993, 660) Ce modèle hypotextuel oral intègre l'invocation auditive et visuelle de Ségou de toute" forme-sens dans la construction [...] spécifique du texte" romanesque. (Id:661)

Tout en étant un écart, un fait textuel discontinu, un signe de l'art, le style circonscrit la doxa ${ }^{2}$ de la stylistique des Ch.B et interprète leurs manifestations linguistiques. Leur expérience esthétique banale singulière donne une sorte d'inflexion à l'émotion et au plaisir, (Cf., Schaeffer, 2015) voire paradoxale. Alors, les Ch.B s'opèrent esthétiquement et s'inscrivent à l'invocation du paradis perdu dont les caractères essentiels dans les rythmes et leurs phénomènes prosodiques, dans les images surprenantes sous ses dimensions sémantiques et stylistiques. 
L'exploration des Ch.B passe sous l'axe théorique de la stylistique littéraire de Jean-Marie Schaeffer ${ }^{3}$, sans négliger l'apport important du pivot épistémologique de la phonostylistique selon Pierre Roger Léon ${ }^{4}$.

Bref, ces deux axes morphologiques et techniques nous poussent à mener notre étude par le biais des vecteurs analytiques suivants:

\section{Les patrons rythmiques (PR) et leurs} phénomènes prosodiques

Les patrons rythmiques "créent la vitalité de la communication expressive" (Pierre Léon, 162) des Ch. B dont les sujets parlant possèdent leur propre expressivité et leur imaginaire linguistique. (Anne-Marie Houdebine, 1982) Les PR provoquent "le court-circuit poétique et transmue le cuivre en or, la parole en verbe. " Dans les Ch.B, la puissance de la mélodie et du rythme sont des forces créatrices. Les PR y "engendrent non seulement la mélodie, mais aussi l'image par son élan itératif et, partant, suggestif, créatif." (J. Derive: 2006) Ceux-ci saisissent ainsi les images de Ségou comme des entités indissociables et s'avèrent le fleuve de Joliba avançant dans les brousses de Ségou.

\subsection{Le rythme syncopé (RS)}

Le chant africain est "l'objet de jeu rythmique concurrentiel." (K. N'Goran, 2009) Condé pourrait le rendre le garant d'une bonne pratique créative de son plume. Le RS, une dynamique d'organisation orale

(Invocation du paradis perdu...) $\quad$ Dr. Amgad El-Zarif 
inséparable de l'étalage de la signification, participe à la spécificité du sens des Ch.B. Il joue également une fonction dans l'interprétation essentielle de la réflexion proposée du corpus de l'étude.

Remarquons que le mode de vie de Ségou est initialement liée à une partie inhérente de la civilisation de Bambara celle du pâturage. La restauration auditive des images du pâturage dépeint une partie de la réanimation $\mathrm{du}$ paradis perdu. À Ségou, "Les hommes étaient amoureux de leurs bêtes plus que de leurs épouses et en chantaient la beauté le soir devant les feux de bois." (SMT:25) Ils chantent:

Ton père est mort, tu n'as pas pleuré.

Ta mère est morte, tu n'as pas pleuré.

Un menu bovin a crevé et tu dis Yoo!

La maison est détruite

$(\mathrm{SMT}: 25)$

Lorsque le chant attire l'attention du lecteur (= récepteur/ décodeur) sur cet amour du naturel, telle que "la vie était rythmée par la saison, les troupeaux allant et vent des pâturages." (SMT : 25) L'image incarnée acoustiquement dans la vigueur et la qualité particulière de la voix reflète excessivement l'attachement de la personnalité à ce monde pastoral. La mort du père ou de la mère est banale par rapport à la perte d'un menu bovin. Le 
chant provoque la surprise sonore à travers l'onomatopée , $^{5}$ qui passe soudainement par le cri aigu longuement modulé Yoo!

Ce gémissement amer, douloureux, algique, poussé en cas d'affliction profonde, fait référence à deux sensations: la première est l'irritation associée au personnage à cause de la perte d'un petit animal dans le troupeau, et la seconde est la stratification consistant à répéter ce monème bambara chaque fois qu'un animal meurt du troupeau. Ce poème nocturne excite la puissance de l'intensité vocale pour augmenter sa souffrance personnelle en trouvant ce menu bovin.

Par conséquent, une répétition sonore intonative forte et violente fait partie intégrante des tentatives de restauration du troupeau au paradis. L'élément manquant du paradis est généralement évoqué par un agent externe au chant; les lexèmes magiques "amulettes", "cornes" et "dents d'animaux" (SMT, 25) protègent les humains et les animaux de la mort.

Dans le chant, les recettes magiques sont encombrées et accélérées, et donnent une forme renouvelée et plus approfondie qui ramène l'âme des parents morts à Ségou. Cette immersion dans des syntagmes nominaux tels que: "écorces fraîches de cailcédrat", "poils de phacochère" et "gouttes de sang menstruel d'une femme avorté sept fois", poudre de coeur de lion séché" créent une connotation mystérieuse et un peu naïve que viennent confirmer des syntagmes évoquant

(Invocation du paradis perdu...) $\quad$ Dr. Amgad El-Zarif 
la sphère primitive et magique de Ségou. Le chant prend ainsi la forme d'une parole rituelle d'une voix très basse, quand le griot, koumaré, chuchote en murmure :

Ke korte, père, mère, ancêtre,

qui es dans la région d'en bas

tu me vois, complètement aveugle

(S.M.T. 46)

Si le chant s'initie par la construction idéophonique ${ }^{6}$ Ke korte, défini par "sa double particularisme phonétique et sémantique" (Dumestre, 1998, 321), il retient comme un trait saillant du chant signifiant en bambara "une dose de ce poison violent qui se lance à distance." Cette construction mélodique à trois syllabes $[\mathrm{ko} / \mathrm{m} \supset \mathrm{r} / \mathrm{t}$ ] correspond au prénom du héros Koumaré composé de mêmes syllabes $[\mathrm{ku} / \mathrm{mar} / \mathrm{re}]$, afin de spécifier avec précision la déglutition lente du poison.

Cette anesthésie acoustique se voit comme stimulus sensuel pour invoquer l'image de ces ancêtres. Une fois que Koumaré a avalé l'appât vocalique, les membres de la famille se sont accrochés dans une accumulation bien organisée: père, mère, ancêtre. La voix "dans sa dimension sonore et originale, dont la voix/voie nous est encore impénétrable. Et l'histoire, à écrire."(Bourlet, 2007, 30)

(Invocation du paradis perdu...) $\quad$ Dr. Amgad El-Zarif 
Ce chant fait donc partie d'un travail de mémoire, d'une promesse probablement fantastique à retenir l'utopie perdue de la mémoire de la famille, des ancêtres, de la mère, du frère, de la bien-aimée, du sol natal ...etc. De plus, la cécité du héros est une condition préalable à l'évocation spirituelle pour révéler un aperçu lumineux à Koumaré quand il se dirige vers son père provoqué dans un énoncé assertif: "tu me vois, complètement aveugle." La parole rituelle est ainsi posée toute entière sous le signe de la remémoration, associée à une tonalité de réminiscence du passé.

Le chant exprimait une représentation épique très authentique $d u$ héros Bambara. Les éloges proviennent généralement d'un événement fort rappelant la fameuse chasse au lion:

$$
\text { Le lion jaune au reflet }{ }^{7} \text { fauve }
$$

Le lion qui délaissant les biens des hommes

Se repaît de ce qui vit en liberté

Corps à corps, Tiéfolo de Ségou

Au plus fort de sa chasse, c'était encore un enfant

Tiéfolo Traoré...

(S.M.T. 66) 
Le chant affleure l'illustration épique de la restauration de la gloire de Ségou, centrée sur "l'éloge du Bambara". L'analyse du RS, ce genre musical privilégié de Ségou, éclaircit "les valeurs éthiques les plus profondes et nous introduit dans le coeur de la structure sociale." (Piga, 2018, 202) Il vise également à renouveler la louange adressée à la classe noble. Ce chant tambouriné diversifie en intensité et en rythme. Le bruit sonore est provoqué par l'intrépide d'un événement imprévu "rappelant la fameuse chasse au lion."

Ainsi, ce patron du rythme au sein du chant se divise en deux types. Premièrement, un rythme syncopé vocal est un fort résultant d'un stimulus énergétique externe quand "le diély frappant leurs Tamani ${ }^{8} . "$ (= $N^{\prime}$ tama en langue bambara), cet instrument primitif s'associe à la diffusion des informations dans la ville. Le timbre catalyseur audio touche les cœurs devant les oreilles pour rassembler les auditeurs autour du chant. Cet instrument sert donc à rassembler très rapidement les gens afin de voir le héros victorieux dont "les femmes sortaient sur le pas des portes, [et que] les hommes (le lion chassé) hélaient." (S.M.T. 66) Deuxièmement, la distribution musicale du chant crée par l'imagination un rythme syncopé interne principalement adopté dans le faisceau de traits phoniques où les constructions syllabiques : cv / cvc sous une forme équilibrée dans certaines combinatoires expressives telles que: reflet fauve [ra/fle/fov], fort de sa chasse [fJr/ do/sa/ fas] encore un enfant $[\hat{a} / \mathrm{k} \supset \mathrm{r} / \varepsilon / \mathrm{a} / \mathrm{f} \hat{a}]$ donne l'avantage à " rythme sonore plus percutant n'est

(Invocation du paradis perdu...) $\quad$ Dr. Amgad El-Zarif 
pas sans rappeler, dans sa composition, certains genres poétiques oraux. " (Bourlet, 2007, 29) Cette accumulation rythmique paraît comme un procédé adhérant aux Ch.B.

\subsection{Le rythme dansé (RD)}

Le RD développe ses enjeux rythmiques d'invocation de diverses images mythiques du paradis perdu. La représentation animale s'émerge encore une fois pour introduire la figure humaine du héros bambara d'une manière pleine d'élégance et de vitalité; ce qui lui octroie un état de sainteté. Condé n'a pas trouvé mieux que la grue couronnée, avec son $\mathrm{RO}$, liée au patrimoine folklorique des Bambaras pour étaler la diversité du RO à travers la répétition du lexème Oiseau en milieu du poème métrique. Celui- ci permet une confession immédiate de la soumission, de l'abstraction du sens de la parole:

\section{Salut grue couronnée}

Puissante grue couronnée

Oiseau de la parole.

Oiseau au bel aspect.

La voix est la part dans la création.

(S.M.T. 76)

Tout en poursuivant l'interaction avec cet oiseau merveilleux, nous découvrons qu'il s'est incarné pas à pas 
phonétiquement et par conséquent rythmiquement dans le chant, simple et rapide.

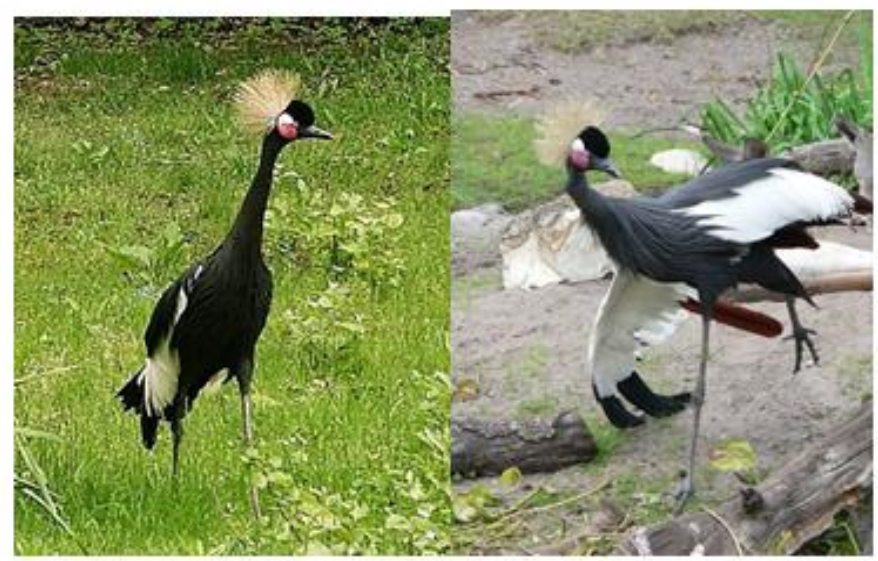

Figure $1^{9}$

Au niveau phonémique et rythmique, le faisceau de traits phoniques crée le trait pertinent phonostylistique. (Léon, 2009,163) Les idéophones ${ }^{10}$ des phonèmes du chant ont ainsi pris quatre niveaux de la distribution musicale:

a- les deux syllabes [gry] et [wa] ont renforcé, l'onomatopée, la simulation phonémique des cris formant le nom de cet oiseau créatif, représentant aux oreilles de l'auditeur,

b- les deux consonnes liquides [r] et [1] largement répandues dans les lexèmes du chant: Salut, grue, couronnée, la (4 fois), parole, part, création concrétisent la créativité lyrique de l'oiseau et sa capacité à être le seul oiseau à infléchir sa voix et son corps, quand il crie pendant la danse surtout nuptiale, 
c- l'épithète postposée couronnée du nom de l'oiseau, avec sa métrique égale cv / cv / cv, [ku/rJ/ne] a pu dessiner avec une grande précision, cohérence et coloration de la forme de la couronne qui orne cet oiseau royal,

d- le chant se composait de cinq vers dont les quatre premiers, des énoncés nominaux d'une métrique égale octosyllabes, décrivent l'état permanant d'agilité, de légèreté et de longueur coordonnée de cet oiseau, pour qu'il se transforme en acte de danse et de chant en même temps. Alors que le dernier vers se présente sous la forme d'un énoncé verbal du verbe copule être; ce vers, composé de décasyllabe, se distribue en deux hémistiches dont chacune se compose de cinq syllabes pentasyllabe: La/ voix/ est/la/ part// dans la/ cré/a/tion. Nous estimons que chaque hémistiche forme une des deux pattes de l'oiseau avec ses cinq doigts acérés, fins et longs pour que les phonèmes du chant mutent complètement en calligraphie dansée et mouvementée dans un spectacle inoubliable, et que le chant devienne les cent pas d'un air à la fois altier et irrité de la grue couronnée. Le chant s'inspire donc sa technique orale des mouvements de la grue; des attaques en pique des doigts imitant les coups de bec, des postures sur une seule jambe, le choix des mots rappelant les battements d'aile.

D'ailleurs, la superposition de la voix charme d'intensité faible s'esquisse sous une grille énonciative à travers la voix féminine de la mémoire acoustique de Naba. La courbe intonative descendante de la restauration

(Invocation du paradis perdu...) $\quad$ Dr. Amgad El-Zarif 
de la voix de la mère Nya s'entend comme une technique magique du chant Bambara qui s'érige en système du discours direct. Faut-il se rappeler/appeler la mère? La mimique de la caresse rythmique, aide le héros exclu au soulagement prosodique. Naba "retrouverait des mots que sa mère lui adressait quand il était tout petit tandis que toute sa vie à Ségou repassait devant ses yeux." (SMT, 107) Nya chantait:

Allons mon bébé

$$
[\cdots]
$$

Qui t'a fait peur?

L'hyène t'a fait peur

Vite, vite, emportons-le à KouliKoro

A KouliKoro, il y a deux cases

La troisième est une cuisine...

(SMT, 107)

Le rythme ternaire expressif Allons/ mon/ bébé "constitue une unité sémantique qu'on appelle "phonostylème." (Léon, 2009,164) L'accentuation ternaire de la succession pas à pas des syllabes crée un vecteur acoustique vers Ségou; elle organise le rythme dansé aboutissant à l'image de la mère/Ségou.

Premièrement, la forme énonciative allons d'un verbe purement du mouvement engendre un dynamisme 
rythmique ascendant au début du chant afin de motiver la mentalité de Naba à s'entamer dans le départ imaginaire. Cette invitation gratuite de la mère gravée dans l'esprit du héros s'offre comme un bonbon délicieux par l'activité bercée du rythme équilibré dans le monème mélodique bébé d'une même syllabe réplétive $\mathrm{cv} / \mathrm{cv}$ [be/be]. L'aspect acoustique du chant renforce cet effet de filiation.

La satisfaction sensuelle rythmique du voyage vers la patrie perdue, mélangée par la force du départ, s'effectue par le verbe à l'impératif de valeur directive emportons en raison de l'identité du préfixe em que par le pronom personnel "le" qui neutralisent le héros égaré dans un univers étrange à lui. La répétition des lexèmes "allons, mon, bébé, vite, à, Koulikoro" bourdonne l'imprégnation momentanée et occasionnée par un RD accéléré du départ.

Le RD suit enfin un état de brainstorming du héros oscillé en trois étapes. Dans la première étape, il est surprenant que le héros ait été informé du plaisir d'entendre la voix de la mère. Ensuite, il se transforme en un rythme intermittent en s'enquérant aux peurs de la petite enfance sous la forme d'intonation soudainement montante de l'énoncé interrogatif Qui t'a fait peur? Sous la forme d'un moteur étonnant, le RD s'accélère à travers la répétition de l'adverbe du parcours spatial en peu de temps Vite, vite. À ce moment, ce rythme dansé syllogistique se transforme en une équation mathématique. À partir du cinquième vers jusqu'à la fin 
du chant, les syllabes s'étendent pour former une aune, se référant à l'ancienne unité de mesure agricole pour le bambara et reflétant le génie agricole de Naba. Ses trois derniers vers de syllabes qui peuvent être divisées en deux aunes (= rythme dansé bilatéral), chacun d'environ 17 pouces / syllabes, nombre d'unités de l'aune. Dans ce rythme prolongé, le décalage spatial hypothétique est apparu dans le syntagme spatial à Koulikoro [a/ku/li/ko/ro] pour dessiner tendrement avec ses cinq syllabes répétées deux fois les deux mains de la mère avec ses dix doigts qui portent l'enfant au sol natal.

Le RD s'abrutit fermement en disséminant l'ennui ou l'apathie de la longue distance du voyage chanté. Sans avertissement, il ralentit pour faire une pause des tracas de long voyage. Il devient indolent, pas à pas, d'une pièce à l'autre. Au fond, le chant schématise son itinéraire sinueux s'inscrivant dans une perspective transitoire jusqu'à à la cuisine.

Avant de se lancer dans le voyage sacré, la mère demande: "Qui t'a fait peur?" Cette question hypothétique aurait également renouvelé et diversifié l'accélération du rythme, et émergé des dépôts des peurs infantiles de l'hyène. La mère a d'ailleurs renvoyé Naba sur la première place de sa vie pour consolider un effacement temporaire de sa mémoire actuelle afin de guérir les douleurs de l'exil. 


\subsection{Le rythme ondulatoire (RO)}

Si Ségou est le paradis, la sortie de ses murailles est l'enfer lui-même. C'est ce qui a frappé Malobali quand il a quitté la ville malgré lui. Ici, le chant se transforme en cauchemar, une voix qui émerge de l'intérieur du personnage pour annoncer de mauvais augure. Le RO du chant devient une voix (un cri) interne qui alerte le héros des dangers qui l'entourent. "Ce cri déchira le demisommeil de Malobali. Il se redressa à demi. La voix répéta: "Sauve-toi, Bambara, sauve-toi!" (SMT, 262) Le chant ci-dessous reflète le mauvais augure de Malobali:

Le serpent qui s'en va

Compte sur les feuilles mortes

Pour dissimuler ses petits.

Toi. Sur qui as-tu compté?

A qui nous as-tu laissés

En t'en allant au pays des morts?

$$
\text { O kou, O kou, O kou }{ }^{11} \ldots \text { ! }
$$

(SMT, 262)

Cette lutte interne jette son ombre sur un $\mathrm{RO} d u$ chant, de sorte qu'elle se transforme en piste du conflit entre le héros Malobali et le serpent; la consonne fricative ou constrictive [s] produit des bruissements de la forme et $\mathrm{du}$ mouvement du corps du serpent quand il touche les

(Invocation du paradis perdu...) $\quad$ Dr. Amgad El-Zarif 
feuilles mortes dans les monèmes serpent, s'en (bis), sur (bis), dissimuler, ses, laissés. La consonne [s] représente d'une part, dans sa propre structure, un rétrécissement des parois, et il fonctionne, d'autre part, à l'anesthésie des nerfs du héros grâce au processus acoustique qui peut donc introduire un effet psychologique inquiétant dans les veines du héros. Une réaction anti-phonémique traduit les gémissements, les cris de Malobali à travers les voyelles [ã] et [ग] dans s'en va, mortes, t'en allant au, morts dont l'aperture, la résonance labiale cristallisent progressivement la panique du personnage pour se souvenir de Ségou. Le bruit plus ou moins consonantique et vocalique assure un rôle de feedback interactionnel (Mareüil, 2014, 10) du chant.

Or, les tentatives essoufflées de sortir de la situation chancelante s'affrontent à la fin du chant, avec la répétition renforcée du lexème bambara calqué $\mathrm{OKou}$. Cette résonnance buccale de la voyelle [u] va directement de l'oreille comme au cœur, pour sceller le sentiment de faiblesse et l'inévitable défaite. La voyelle fermée [u], la plus rapprochée du palais, à l'extrême du chant, faisant un redoublement expressif, une homophonie avec le cou [ku] en français, suivie de trois points de suspension et d'un point d'exclamation, se transforme en étreinte pour étouffer le héros au strict nécessaire. Le RO portraiture ainsi l'accablement du héros à escalader les murailles de Ségou. 
D'ailleurs, certaines voyelles s'avancent en s'enroulant autour du cou du héros et en resserrant progressivement ses anneaux provoquent l'asphyxie vocalique. Cette pression phonétique et rythmique crée une variante connotée et subjective du terme d'une onomatopée agaçante et troublante $O \mathrm{kou}, \mathrm{O} \mathrm{kou}, \mathrm{O} \mathrm{kou}$. Celui-ci entraîne une fin pathétique à tous les rêves de regagner Ségou. En l'occurrence, l'exclamation au dernier vers du poème se réfère à la tradition orale bambara qui reprend la voyelle typographiquement écrite en majuscule à la fin du poème. Le statut d'interjection laudative se déduit par le fait que l'expression calquée $O$ kou! crée une clôture angoissée et morne au thème de la mort dont l'odeur se répand dans tous les vers. Condé plante ce motoutil $O$ kou au bout du chant. Si le RO fait un bruit de fond auditif, l'effet global est évidemment différent : l'écrivaine/poétesse " ne cherche pas tant à mettre ce motoutil en évidence qu'à l'utiliser comme un tremplin " (Lemaire, p. 373), il sert toutefois pour arriver au but thématique du chant: le départ vers la mort.

Par ailleurs, le RO suggère le deuil, ce tumulte intérieur de Diémogo " [qui] se jeta dans la poussière" de la mort du chef de la famille, Dousika. Cette chute spontanée et ce fléchissement des genoux revêtent les bouleversements de l'âme. En ce moment, le chant est un sanctuaire inévitable pour tirer l'âme d'un état de grande tristesse de la désintégration de la famille Traoré. Le RO prend une forme de gémissements intermittents dans son

(Invocation du paradis perdu...) Dr. Amgad El-Zarif 
mouvement circulaire où "Tiétigui Banintiéni se mettait à tournoyer autour de lui "Diémogo" en criant":

\# Koro, ton unique bâton d'appui

$$
[\ldots]
$$

Quand tu auras encore besoin de soutien

Vers qui iras-tu à présent?

$(S M T, 143)$

Le RO formant l'expressivité de réincarnation de l'image de Dousika par la combinatoire phonologique, peut se retrouver dans le phonostyle snob (Mettas, 1966) telles les occlusives dentales fricatisées ( Léon, 2009,163) de trois consonnes [t] dans tu (5 fois), soutien (bis), [b] dans bâton, besoin (bis) et [p] appui, apprennes, présent. La mime du sens de vives condoléances provoque une pression aux oreilles de Diémogo comme celles du lecteur par la suggestion acoustique de schématisation consonantique dans le chant. La voix de l'ami, Tiétigui Banintiéni, aussi que ses gestes, pénètrent la peau, tout le corps de Diémogo réactivé par le chant. L'âme de la personnalité affectionne le RO plié opératoire du concept des consonnes. Le chant admet ainsi les mécanismes des forces enchaînées afin qu'il autorise " la réincarnation dans le corps" : (SMT, 143) du frère mort.

Parallèlement, la consonne occlusive [t], dans $t u$ (5 fois), soutien (bis), prononcée en appuyant la langue 
contre la rangée inférieure des dents, fréquemment répétée dans le pronom personnel $t u$, souligne que Diémogo a accepté l'absence morale de soutien du frère décédé. Tandis que les autres consonnes occlusives [b], [p], formées par le desserrement brusque des lèvres, dans bâton, besoin (bis) et appui, apprennes, présent s'efforcent non seulement d'imiter leur forme calligraphique de soutien mais aussi de sonner aux oreilles de Diémogo afin de compléter l'évocation de l'image du frère dans le chant. Le processus dynamique du rythme ondulatoire se présuppose pour "suivre l'âme de Dousika dans son voyage jusqu'à la demeure des ancêtres." (SMT, 144)

Cette expérience orale se déplace avec une grande spontanéité aux phonèmes du chant pour taquiner les sensations de l'auditeur; cette incroyable capacité retentissante navigue dans les profondeurs des sentiments tristes du héros. Un RO ballant ou oscillant se répartit en mouvement de balancement dans les quatre premiers vers. Immédiatement, la répétition est souvent utilisée comme un mode d'injection auditive poussé à la ligne de musique externe "des battements des femmes. " D'ailleurs, le même vers en syllabes très proches "J'irai au marigot, ma mère!" (SMT, 430) s'interprété en l'honneur de la mort de Tiékoro, héros décédé.

De ce fait, de la simple interférence itérative à la mélodie réelle, les limites vocales semblent assez spécifiques. Pour se limiter aux cris de deuil, ces

(Invocation du paradis perdu...) $\quad$ Dr. Amgad El-Zarif 
expressions rythmiques imposent une cadence régulière fréquemment associée au rythme présenté par le facteur humain sonore: les mains des femmes ayant une courbe mélodique naturelle issue des travaux ménagers pour devenir en fin de compte l'un des instruments folkloriques émotionnels.

Le RO évolue tout en aménageant le voyage du héros en route en vue de ranimer l'univers mythique comme un monde céleste souvent nanti de glorification. Au-delà de cette description, les phonèmes développent l'allocation du deuil, auquel la distribution de [m], [r] et [o] s'intègre à l'état morne des femmes gémissantes. Or, ces trois phonèmes représentent une trinité de hurlements où la voix $[\mathrm{m}]$ évoque le bourdonnement des femmes; les deux phonèmes suivants forment la dernière syllabe de (Koro); le prénom du héros, et ils deviennent la momie du corps du héros découverte aux tumultes cris plaintifs féminins.

Cette forme d'opéra lyrique confère au chant une forte résonance partout dans la ville pour annoncer la mort $\mathrm{du}$ héros. Le répertoire rythmique cantonne les voix douloureuses des femmes pleureuses dans le contexte adéquat. Elles chantent intensivement possédant un registre vocal oral résolu s'harmonisant avec leur rôle quasiment cérémoniel concomitant avec le mécanisme du tapotement des mains. 
Cependant, le chant de la mort d'un personnage hostile, Pemba, peut se transformer en une réjouissance populaire, joie collective; une chanson ironique circulait:

Pemba, tu es le constructeur des choses, Fado $^{12}$, toutes les choses de l'univers Sont en son pouvoir. Celui-qui-s' assied-// sur- la-peau-de-bœuf $f^{13}$ Avait oublié cela!

(SMT, 465)

Et les femmes faisant tomber leurs pilons dans les mortiers fredonnaient (chanter à mi-voix; sans articuler les paroles) en chœur :

$$
\begin{gathered}
\text { Celui-qui-s' assied-//sur-la-peau-de-bouf } \\
\text { Avait oublié cela! }
\end{gathered}
$$

(Idem)

Certaines caractéristiques collectives de l'oral portent un marqueur rythmique discriminatoire à travers l'inhérence des " pilons" et des " mortiers" tout au long du chant avec les femmes qui "faisant tomber leurs pilons dans les mortiers fredonnaient en chour." Comment le RO du mort peut devenir " une vengeance divine" ?

(Invocation du paradis perdu...) Dr. Amgad El-Zarif 


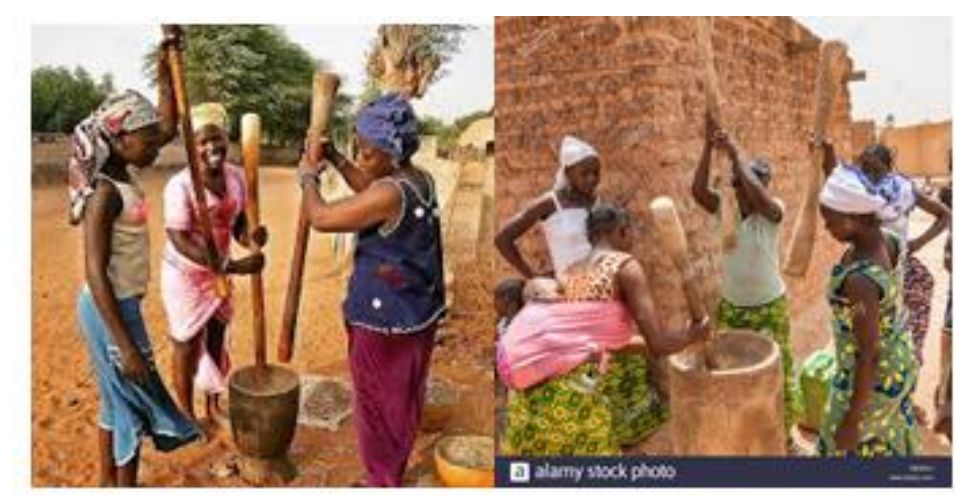

Figure $2^{14}$

Ce chant axé sur "la mémoire développe des liens particuliers entre le visuel et l'écriture" qui "émaillent le tissu narratif, auquel ils accordent une grande expressivité de par leur valeur figurative." (López, 412) Il accrédite également "la mémorisation et l'intériorisation des normes collectives" des Bambara, et il demeure la "réponse reconnue comme juste est celle fixée par la tradition." (Hecquet, 2009, 4) Les femmes rythment ces vers par un refrain (du quatrième au cinquième vers) en les terminant à chaque fois par le refrain itératif avait oublié cela!, divisant aussi le quatrième vers en deux hémistiches pour planter l'insistance rythmique d'un désir ardent de vengeance de cette mort par l'effritement, le meulage de Pemba dans le mortier par le pilon. La collectivité du chant donne une forme d'écho d'une concession à l'autre dont la " chanson, c'est insaisissable comme l'air." Le pilage opiniâtre revêt des sonorités particulières des consonnes [p] et [b] dans Pemba, pouvoir, peau, bouf, oublié. Ce RO robuste et

(Invocation du paradis perdu...) $\quad$ Dr. Amgad El-Zarif 
ininterrompu donne aux paroles des femmes leur impact inamissible.

La voix des femmes regroupe " un bruit de fond et une stimulation sonore qui prend une acuité plus grande car elle fait appel aux formes profondes de l'imagination collective" (Zumth, 2008,169) de Ségou. Le chant en acquiert une force et un rythme frappants tout en créant une mélodie originaire du chant et en conférant la richesse et la complexité réflexive de la mort.

\subsection{Le rythme nostalgique (RN)}

Le RN apparaît à partir d'un groupe d'éléments phonémiques et parfois syntaxiques dans un certain nombre de chants relié aux thèmes à la restauration du paradis perdu, tels que: l'héroïsme, le mariage, la chasse et la maternité. Par conséquent, les images audio se réunissent pour unifier le type distinct de l'une des formes du rythme au chant.

Le RN participe exprès au rappel des exploits mémorables dans la brousse de Ségou où les griots se précipitent vers le Tiéfolo en l'appelant Chasseur, chasseur:

\section{Chasseur, chasseur}

Si tu vantard, je ne vais pas te louer

N'est-ce pas toi qui examines l'éléphant

Poursuit le buffle

(Invocation du paradis perdu...) $\quad$ Dr. Amgad El-Zarif 
Et fais disparaitre la girafe

Au pelage couleur de soleil?

Chasseur, chasseur, si je te chante pas

Qui seras-tu?

N'est-ce pas la parole qui fait l'homme?

(SMT, 343)

Les griots ${ }^{15}$ ont le pouvoir gnomique et oratoire, la possibilité de capter les notes de leur mélodie; ils s'étendent doucement sur le thème de (la louange du prince Tiéfolo.). Toujours le monème Chasseur pivote sur le RN, restauré en chantant. Ceux-ci manipulent la courbe intonative par de déviations syntaxiques axées sur des mouvements mélodiques ascendants: négative et interrogative. Cette duplicité rythmique jongle Tiéfolo dans les brousses des énoncés cadencés.

Pour les griots, le RN architecture un réseau à échelettes variées. En plus, cette désignation disséminerait l'enthousiasme dans les veines du héros pour restaurer les gloires de la chasse dès son enfance en se rappelant "le lion qu'il avait tué à dix ans." (SMT, 343)

Les énoncés au conditionnel chavirent le héros dans le cas d'embarras de chasse habileté. Sans ces griots, celui-là ne s'avance pas vers la brousse. La phrase complexe conditionnelle l'oblige à se soumettre aux

(Invocation du paradis perdu...) $\quad$ Dr. Amgad El-Zarif 
conditions requises des griots à travers le $\mathrm{RN}$ accéléré du mouvement mélodique bilatéral équilibré dans le nombre des syllabes du chant:

Sil tu/van/tard,// je ne/ vais/ pas/ te louer

Sil je tel chant/ pas//

Quil se/ras/-tu?

Le grimpement du RN se fait en fonction de la réponse de deux clauses abusives des griots. Dans le premier cas, la négation postposée je ne vais pas te louer renforce la tension du héros. Dans le second cas, l'interrogation oratoire, Qui seras-tu? marginalise le rôle du héros dans la chasse.

La complexité syntaxique de la deuxième phrase, maintenue dans le chant, s'étend du deuxième au cinquième vers et affecte le héros dans un état de harcèlement auditif sévère et compatible rapporté à l'effort débrouillé dans la chasse des animaux féroces. Vient ensuite l'avant-dernière phrase qui commence par l'appel répété Chasseur, chasseur, ce qui suscite l'espoir et octroie une sorte de sûreté à la poursuite du voyage de chasse, par le biais des modalités rythmiques des griots déjà identifiés plus haut: si je te chante pas.

Le chant annonce alors le passage d'une instance énonciative: un changement de point de vue, ce qui est le cas de l'épanorthose qui permet de faire surgir la voix d'un narrateur narrant (le griot) à propos de la narration, et

(Invocation du paradis perdu...) $\quad$ Dr. Amgad El-Zarif 
suscite un commentaire. Nous passons ainsi de "la voix $d u$ narrateur narré à celle du narrateur-scripteur, qui, avec la première personne (Je), commente la concordance de l'image avec l'occurrence." (Rouayrenc, 2015, 7)

Le dernier énoncé du chant "N'est-ce pas la parole qui fait l'homme?" annonce fidèlement le rôle animateur du chant dans l'enthousiasme du héros à travers un RN escaladé pour télécharger le chant de trois cellules combinées: assertion, négation et interrogation, qui rendent Tiéfolo un gibier à poil dans les griffes du chant. Cette magnifique manipulation rythmique esquissant l'expédition de chasse impassible dessine une peinture murale contre les murailles de Ségou, un témoin de l'aspect reviviscent du chant de louange. Celui-ci transcrit le "folklore de la chasse; mentalité et rites des chasseurs bambara [...] Mythes, rites et récits initiatiques" (Arseniev, 2341 )

Le RN du chant émerge ensuite un sentiment sincère pour ranimer la mémoire d'une femme chérie. L'expressivité morale du texte narratif réside davantage quand Condé envoie son jugement sain en exprimant que "l'amour ne peut se confondre avec un autre sentiment." (SMT, 447) Condé particularise l'extension accordée à ce genre d'énoncés émotifs dans lequel Ayisha voyait qu'Alfa "était le maître qu'elle s'était choisi." Le griot Amadou Sandji commente ce souvenir agréable, il "chantait un chant traditionnel d'épousée":

Il a bien raison, le roi, de nous battre. 
Il bat le tambour royal pour nous en faire entendre le son,

$$
\text { [...] }
$$

Les femmes reprenaient en chœur le refrain:

Il a bien raison, de nous battre, le roi.

(SMT, 447)

Condé reformule le $\mathrm{RN}$ du chant du louange/amour accentué par la mise en relief d'un instrument musical primitif: le tambour royal. Son émission sonore procure aux oreilles les différents dons du roi/batteur. Celui-ci provoque les humeurs évoquées du chant par une grille des phonèmes, des verbes et des syntagmes nominaux favorisant le véhicule sonore de la ré-existence du roi bambara. Des consonnes et des voyelles sont également distribuées pour amplifier la répulsion les mélodies du tambour royal. Il s'agit d'introduire des jeux consonantiques dans [R], [p],[f],[k],[J], et vocaliques dans $[u]$ et $[\varepsilon]$ qui s'établissent sur l'effet produit par des zones articulatoires fermeture/ouverture, orales/nasales du chant.

En outre, l'interprétation minimale des sons nous conduit à présumer que les consonnes $[\mathrm{R}]$ et $[\mathrm{b}]$ enracinent l'idée d'un esprit royal incroyable et condensé par les termes: roi(bis), royal, battre, destriers. Par ailleurs, d'autres consonnes telles que [p], [f], [k], [J] en: pour (3f), peau, nuptiales, fait, faire (4f), femmes, Kola, roquer, achète (bis), chevaucher exposent le contenu de

(Invocation du paradis perdu...) Dr. Amgad El-Zarif 
la boîte de cadeaux matériaux accordé par le roi, satisfaisant tous les goûts. Les deux prédicats roquer et chevaucher, par leur dimension onomatopéique, concrétisent dans le cas de roquer le mouvement fort et sonore des mains en choisissant le Kola, alors que le prédicat chevaucher glose le mouvement réciproque de monture, de changement sémantique entre le cavalier et le destrier (cheval de bataille). Tandis que les voyelles [u] et [E] dans nous (5f), claire, achète (bis) reflètent simultanément l'état de surprise extrême et le bonheur écrasant de l'auditeur de l'abondance de cadeaux présentés par le roi.

Et encore, les phonèmes de la percussion royale démontrent non seulement le pouvoir royal, mais les dons à tous, chaque fois que le ton du tambour change, une nouvelle sensation atteint l'auditeur d'une nouvelle générosité déterminée par les termes du chant soigneusement choisi " pour nous en faire entendre le son." La perception nostalgique du mariage renforce cette fonction identificatrice (Léon, 2009, 167) du RN.

La variété prosodique donne du dynamisme au langage tambouriné d'une spécificité tonale royale. Le tambour ravive l'épopée de Ségou sous une forme d'écriture lyrique illustrée selon la figure ci-dessous: 


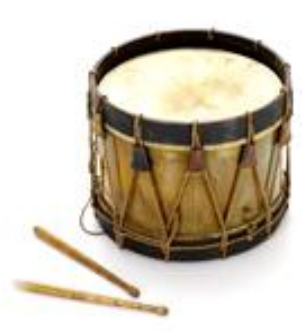

Figure $3^{16}$

Par le biais de ce tambour royal, le griot, Amadou Sandji, déclare une annonce proche à l'héroïne Ayisha et un message lointain au lecteur: le tambour royal correspond un RN particulier et variable au cours du développement du chant des cérémonies de louanges, de mariage, de mort, etc. Le RN revive langage bambara tambouriné, une production spontanée de la structure syntaxique et lexicale spontanée.

En fait, les syntagmes panégyriques tels que tambour royal, femmes à la peau claire, chambres nuptiales, etc. sont récités de la part des musiciens qui, "dans le chant et dans la récitation ne se séparent jamais de leur instrument de musique." (Piga 2018, 203) Une référence constante est faite "au seuil de la concession des Traoré." (SMT, 12)

Le héros éprouve des souffrances physiques d'un besoin de l'invocation de l'image de la mère. Le chant est le plus souvent fusionné dans un moment urgent des événements, Siga "était las. Toute la journée, sous le

(Invocation du paradis perdu...) $\quad$ Dr. Amgad El-Zarif 
soleil, [...] il était retourné à Ségou [...] auprès de Nya. Nya, le seul être [...] chéri. Il la sollicitait:

Mère chérie

Mère qui donne librement tout ce qu'elle possède

Mère qui n'abandonne jamais le foyer

Mère, je te salue

L'enfant qui pleure appelle sa mère

Mère chérie, me voilà!

(SMT, 95)

La systématisation des traits intonatifs émotifs (la peur de Siga) et de l'attitude attendue de la mère (tendresse/coquetterie) s'émerge par le fonctionnement des éléments expressifs combinatoires. Le chant met l'accent sur la prière pressante de Siga afin de procurer l'image de la mère, Nya, à travers un RN personnel.

Le primat de la prosodie (Meschonnic, ...,117) se voit dans la forme énonciative du chant. Il s'agit d'un désir ardent de provoquer l'image de la mère lorsque Siga joue avec cette notion du primat précisé dans le bêlement de Siga à travers la consonne $[\mathrm{m}]$, répétée dans le monème Mère, et son chevrotement onomatopéique animale par la consonne [J] dans l'épithète postposée d'affection entre les intimes, chérie. Ces deux consonnes impliquent 
acoustiquement un signal phatique de plus en plus fort, avec la "connotation de signifiant voulant dire." (Léon, 2009, 167) La présence de la consonne [m] en tête de tous les vers, suivie par la consonne [J], crée une suprématie et dispose d'une connotation la primauté archéologique. Celle-ci crée également un souffle amoureux permettant d'identifier le locuteur/Siga.

De surcroît, la répétition vocale inquiétante de l'hypothèse peint un certain état de gémissement et de mendicité utile de la transforme en chevreau qui a perdu sa mère dans le pâturage de Ségou. Mais l'enfant réussit-il à convoquer l'image de la mère disparue? Certes, les indicateurs énonciatifs se réfèrent à l'acte direct de force illocutoire performatif opératoire d'affection et d'importunité " Mère, je te salue," la fonction mimétique se trouve dans cette situation d'énonciation singulière où " l'émetteur [Siga] et le récepteur [Nya sont] présents l'un à l'autre. » (Cauvin, 1980, 42)

Siga, un partenaire actif, élabore ses propres perceptions du sens d'une expression de salutation manifestée par la présence de deux pronoms d'énonciation je (Siga), et tu (Nya). Les consonnes [p] et [1], dans les deux verbes pleure et appelle étayent une échelle liquide des consonnes de béguètements pour entrer discrètement dans la ville. En franchissant spirituellement murailles de Ségou, Siga se trouve sur les pieds de sa mère chérie en réalisant une proximité physique; il a alors proféré son dernier acte d'énonciation de sentiment de bonheur 
intense: "Mère chérie, me voilà!". Le RN caprine rend réelle et effective la contiguïté entre la mère et son enfant.

D'ailleurs, le RN crée la soi-disant nostalgie par la musique de l'espace, cette dernière devient un stimulus acoustique de l'image perdue. Siga "songe [à Ségou = à sa mère] déjà quand les femmes entonnèrent une petite chanson à la fois ironique et tendre:"

Le vent soufflait et la pluie menaçait

Le bimi vint s'asseoir sous un arbre, Pauvre bimi!

Il n'a pas de mère pour lui apporter du lait

Pas de femme pour moudre son grain

Pauvre bimi!

(SMT, 427)

Dans ce contexte, la musique naturelle émane de l'anémophile du vent représenté par [v] [et [s], mixé avec l'ombrophile de la pluie dans [p] [b] dont le chœur musical des femmes qui "étaient accoutumées à la railler, à l'agréable dans les échanges aux sous-entendus chargés de sexualité." (SMT, 427) Le vent, la pluie et les femmes fournissent un orchestre convenable à l'évocation

(Invocation du paradis perdu...) Dr. Amgad El-Zarif 
de l'image de Ségou ayant un lien très étroit à l'enfance ou à l'adolescence du héros.

L'ornement sonore du RN d'un mariage élabore une organisation et une sonorité poétiques cédant deux formes: une forme collective du chœur et une autre individuelle du poète Amadou Sandji qui configure la spécificité d'un tel thème: le mariage à Ségou. Celui-ci, élément folklorique harmonise le $\mathrm{RN}$ du chant $d u$ mariage:

Que ce mariage soit heureux!

Qu'il en sorte pieds et mains!

Que dure le feu de cette union!

(S.M.T. 281)

Encore une fois, la collectivité du chant s'accélère sur une vivacité sonore et englobe la joie exceptionnelle du mariage. Le chœur a la charge d'un double écho comme cri de joie apportant le bonheur au cœur nouveau marié.

Comme un bon rêve, l'accent est mis sur la mémorisation de la fête de mariage. Alfa entendait la voix unique du poète Amadou Sandji accompagné par le chant modulé de la flûte ${ }^{17}$ :

Plein de ventres, la paix à moi me comble.

O mes femmes nombreuses, mes fils nombreux

(Invocation du paradis perdu...) Dr. Amgad El-Zarif 
Moi, j'ai campements nombreux

Et nombreux villages serviles

(SMT, 446)

La flute, instrument monoxyle primitif de six trous, développe le chant modulé qui s'harmonise avec les six énoncés oraux du chant, articulés orthographiquement par une virgule. Un accompagnement instrumental préconisé pour le poème; ce qui renvoie plus à l'audition qu'à la lecture, et suggère un contexte d'énonciation orale.

Figure $4^{18}$

Cet instrument engendre son expressivité ascendante de la progression du RN varié à chaque énoncé chanté. La douce pression du poète griot avec son expiration détendue et paisible précise que la moindre différence dans la prononciation est immédiatement perceptible par l'auditeur (=récepteur, destinataire, le décodeur.) Les consonnes organisées à l'ordre [r] dans ventres (= troupes d'animaux), [l] dans comble, [f] dans femmes, [s] dans fils [k] campements [3] dans villages représentent les traits de la richesse d'Alfa où le chant

(Invocation du paradis perdu...) $\quad$ Dr. Amgad El-Zarif 
maîtrise non seulement la qualité de l'émission, mais aussi la mise en relief du RN liée aux énoncés chantés.

La diversité rythmique construit la première et la dernière syllabes en trois monèmes créant un rythme binaire et manipulant les sentiments d'Alfa créant un lien d'intimité ébranlé du signifiant au service du signifié selon Meschonnic.

Quant au moyen vocal, les [r], [1], [f], [s] [k] [3] engendrent un mode particulier de la performance, et "se situent (du point de vue acoustique) au niveau, soit $d u$ [poète], soit du chant." (Zumthor, 2008, 184) Il parait que le RN a deux registres distincts: 1'un utilise des ressources vocales alors que l'autre émet les sons conformes aux diverses règles linguistiques et parfois esthétiques de nature conventionnelle.

\section{L'étrangeté des images dans les Ch.B}

Les $\mathrm{ChB}$ " chargé(s) de connotations [...] lié(s) à tous les jeux que permet le langage, [...] tendent à renforcer, à leur racine même [...] le lien collectif ; leur force persuasive provient moins de l'argumentation que du témoignage." (Zumth, 170) Le roman grouille de l'esthétique du chant qui vise à rendre compte d'une étrange africanité, il s'est alors agi "d'illustrer un attachement à une terre, à une culture." (Kabuya, 2013,16) vu dans les points suivants: 


\section{a. L'envers du décor de Ségou}

Les $\mathrm{ChB}$, forme de reconnaissance et une galerie de l'espace de Ségou, réalisent la découverte illusoire de cette ville. $\mathrm{Ce}(\mathrm{t})$ lieu / espace prestigieux crée un encouragement d'une enquête imagée ou visuelle. Le reflet d'un cheminement part du reportage chanté en utilisant des codes d'écriture photographique : le paysage et le portrait.

En chantant, les références connotées ont évolué l'envie de dévoiler le réel sous les apparences et d'aller fouiller derrière les murailles de Ségou.

Pour ce faire, cette réalité par la radiographiel la figurabilité figure ce discours ludique et poétique. Les chants passent comme des presse-boutons du photographe qui incarne l'historicité de cette ville.

Dès l'incipit, le roman semble avoir une construction plus variée, dans laquelle la situation initiale produit un brassage langagier unique par un chant des griots qui met le lecteur au début de la route afin d'évoquer ce décor imagé de Ségou, quand les griots chantent:

Ségou est un jardin où pousse la ruse. Ségou est bâtie sur la trahison. Parle de Ségou hors de Ségou, mais ne parle pas de Ségou dans Ségou.

(SMT, 11)

(Invocation du paradis perdu...) $\quad$ Dr. Amgad El-Zarif 
Le premier paragraphe du roman, un chant monotone, dirigé à Dousika, le noble de la Traoré, ne lui prête aucune attention; celui-là a déplacé ses sentiments dans un état d'appréhension et de tenace; Condé l'a associé à un sens physique: la nausée d'une femme enceinte, accordée à l'omniprésence de la puissance sexuelle.

Ajoutons que la réincarnation de l'espace de l'habitat est le signe figuratif de la femme-terre qui "entraîne un jeu particulier [...] des dispositifs optiques qui se réfléchissent les uns sur les autres comme par un effet miroir." (López, 420) Ségou est un rêve qui obsède Dousika. Ce rêve peut-il devenir une réalité? Les termes allégoriques et figurés du chant annoncent le faux-espoir de faire revivre le paradis de Ségou et le lexème espoir vient de dépeindre Ségou comme un jardin en étroite synonymie avec le sème paradis.

L'analogie corrompue de la métaphore filée est graduée parce qu'elle se succède précipitamment lorsque les lexies ruse et trahison à connotation négative accélèrent la description de ce jardin. La valeur sémantique et architecturale des prédicats pousse et bâtie se détruit. Ceux-ci se réfèrent très précisément aux aspects matériels de la civilisation du Ségou, fondée uniquement sur l'agriculture et les travaux de construction, afin de montrer que la trahison et la ruse ruinent cette œuvre.

Par ailleurs, les énoncés constatifs du chant gagnent une profondeur enracinée au cœur de la ville, secouant

(Invocation du paradis perdu...) $\quad$ Dr. Amgad El-Zarif 
Dousika dans sa lutte pour en restaurer la prospérité. Cette lutte morale épique trouve son écho dans la répétition ennuyeuse de Ségou (six fois) pour injecter à tous les côtés du personnage une drogue psychotrope, qui lui donne l'illusion du retour de l'âme (= esprit) dans la ville.

De même, l'opposition localisatrice entre hors \# dans crée une horreur foudroyante dans le cœur du lecteur et du personnage, et un terrible silence qui coupe le sens négatif du prédicat parler, tantôt l'impératif affirmatif tantôt à l'impératif négatif en vue de rythmer la méthode orale du chant en général, et poursuivre gloutonnement et tranquillement la trame des chants épiques en particulier.

La référence à Ségou, cet espace symbolique, soutient l'idée que l'invocation de la sphère poétique devient une source d'inspiration privilégiée pour Condé. Pour Baumgardt et Derive (2005), le chant de l'épopée de Ségou développe le récit collectif des Bambaras qui construit, non seulement une identité idéologique ou culturelle, mais encore une destinée d'immortalité.

D'ailleurs, le chant amènera à la ligne d'arrivée; l'accès au paradis perdu, une simple construction architecturale, qui s'inscrit dans des rêves innocents de l'enfance attachés au sens du goûter. Le dernier lexème d'un poème à la page 107, cuisine, est l'une des clés de l'envers de ce paradis: la nourriture dans l'enfance créant un lien solide entre la mère et la mémoire de l'enfant essentiellement assimilée au plaisir sensuel de la nourriture, qui sera interprété dans des poèmes ultérieurs

(Invocation du paradis perdu...) Dr. Amgad El-Zarif 
sous forme de certains aliments propres aux bambaras tels que tô ${ }^{19}$ ou patates douces.

Voilà pourquoi la sensation établit l'échelle sémantique disposée exclusivement du chant de la mère qui traverse vers sa finalité bien imprégnée par l'abondance des sens de goût et de sentir. Ceci est concrétisé dans un seul lexème bousculé à la fin du chant cuisine, suivi par trois points de suspension afin de dessiller les yeux du lecteur à tous les plats préférés à l'enfance. Ainsi, le rôle de la mère résume une partie de la pyramide des besoins de Maslow qu'on pourrait résumer selon le diagramme ci-dessous:

\begin{tabular}{|c|c|c|}
\hline Besoins humains & Type & Mots dans le chant \\
\hline Les besoins physiologiques & Manger & cuisine \\
\hline \multirow{2}{*}{ Les besoins de sécurité } & Un abri: (logement, maison) & KouliKoro, deux cases \\
\cline { 2 - 3 } & $\begin{array}{l}\text { La sécurité physique contre } \\
\text { la violence, les agressions }\end{array}$ & L'hyène t'a fait peur \\
\hline Les besoins d'appartenance & $\begin{array}{c}\text { Le besoin d'aimer et d'être } \\
\text { aimé (affection) }\end{array}$ & mon bébé \\
\hline
\end{tabular}

De temps à autre, le chant est un point de repère autorisant au héros de s'orienter dans un espace foisonnant du danger. Les traits sémantiques pessimistes font aussi retentir les cris de la mort aux oreilles du héros tout en étant quand-même une sonnette d'alarme qui mène à la transformation spatiale. Condé a choisi son déictique temporel avec précision, c'était l'aube et également le stimulateur sonore externe "le ressac de la mer" (SMT, 430). La configuration mimétique animale à travers le 
lexème serpent a pleinement acquis de mauvais augure pour bien diffuser ses termes empoisonnés du sème /mort/ dans quatre données sémantiques.

Le chant a également choqué l'auditeur avec son étrange représentation spatiale, surtout en ce qui concerne le sème /mort/.

J'irai au marigot, ma mère!

Un mauvais oiseau m'a adressé son chant!

$$
[\ldots]
$$

Les femmes pleurent,

Les femmes se lamentent,

Car leur grand cultivateur s'est couché!

(SMT, 430)

Il est à remarquer que le chant sème le doute cette fois lorsque l'écrivain/poète met un lexème fluvial Marigot au début du poème, destiné à occuper la pensée de l'auditeur: le sème Marigot a des dimensions géographiques fluviales indicatives, se référant à cette partie morte d'une grande rivière; ce qui souligne quatre interprétations possibles:

Premièrement, le héros se transforme en partie sèche et inutile de Joliba. En outre, Marigot pourrait être les tombes funéraires des princes Bambara pour dissiper 
l'image d'une région insalubre et marécageuse qu'évoque Marigot.

Deuxièmement, le marigot se joint au syntagme nominal mauvais oiseau dont l'épithète antéposée mauvais, un quasi-morphème possède de valeurs connotatives néfastes à la mort des héros bambaras. Cette dualité sémantique consolide le sentiment du pessimisme de la situation funéraire, non seulement avec le corbeau rentrant survolant marigot, mais aussi avec le cri prolongé, adressé tout à la fois vers le personnage et l'auditeur. Cet oiseau sombre accorde pareillement des connotations sonores et chromatiques: sa voix est une proclamation d'une mort royale dans la tribu, sa couleur noire est un signal du deuil porté par les femmes bambaras. Ainsi, le chant s'inscrit dans le tissu narratif "pour peindre à travers ses signes un imaginaire riche comparable à celui d'une création picturale." (Pagán López p.420).

Troisièmement, le parcours sec du Joliba ${ }^{20}$ s'est tourné vers l'itinéraire du convoi pour faire les adieux au héros décédé. Ce voyage a amplement assaisonné les prédicats dans un assemblage textuel précis pour peindre les cérémonies funéraires. Le prédicat "irai", au futur simple, répété deux fois, indique une détermination précise du chemin vers le marigot, ainsi que l'autre prédicat m'a adressé ", au passé composé, également répété deux fois, suggère les cris rauques du corbeau, le mauvais oiseau, approprié à l'angoisse terrifiante du

(Invocation du paradis perdu...) $\quad$ Dr. Amgad El-Zarif 
voyage de la mort. Les deux prédicats successifs au présent pleurent et se lamentent connectés au gémissement artificiel pendant les funérailles. Ceux-ci sont à titre d'exemple mis au présent de l'indicatif pour exécuter une synchronisation vocale continue tout au long du cheminement funéraire.

Quatrièmement, La construction formulaire bambara décalqué "grand cultivateur" a été choisie de manière qu'il soit également associé au marigot. Somme toute, le dernier vers relate son lien de causalité: Car leur grand cultivateur s'est couché! Il s'agit d'une mise en relief par le connecteur de cause car. Le chant atténue ce voyage pénible par l'usage euphémique du prédicat "s'est couché" au lieu de "est mort". L'alliance de parole spontanée concrétisée dans l'arrangement chronologique des prédicats du chant: passé composé, présent, futur, revêt la forme d'une fable mythique. Ce récit à base d'imagination est doté d'un ressort narratif se gravitant sur les murailles et les tombeaux de Ségou pour résister aux fluctuations de l'histoire, et pour que le chant soit la voix hésitante qui ravive le paradis perdu.

Condé nous communique le titre du roman par une comparaison sensuelle étrange :" Mais Ségou! Ségou est entourée de murailles. C'est comme une femme que tu ne peux posséder que par la violence." (SMT, 284). Et cette comparaison entre Ségou et une femme incarne le vif désir du retour à Ségou/mère, et confère au chant ses conséquences connotatives antagonistes et violentes. 
L'intensité graphique du chant se développe à l'aide de la diversité apparente de la réincarnation de la chasse dans les prés de savane; cet espace exotique pour le lecteur occidental, du trait sémantique opposé / gigantisme / de la macrophotographie, de l'élément humain opposé au / nanisme / des éléments bestiaux de l'image lyrique.

Le lexème Chasseur émerge la présence humaine répété quatre fois dans le chant, alors que le mouvement animalier accéléré dans la brousse provient de trois énormes animaux (éléphant, buffle et girafe), qui, en fait, possèdent suffisamment de pouvoir pour écraser le héros. Le noyau sémantique/chasse à poils/ se réunit pour octroyer aux animaux une force double et spécifique de qualité de chasse. Celui-ci revêt la sauvagerie qui se rapporte généralement aux croyances des bambaras de métamorphose en animaux.

Les trois prédicats: examine, poursuit, fais disparaitre, font ressentir la violence de l'action de s'emparer, car ils soutiennent le bien-fondé de l'hypothèse de la bizarrerie de l'image. Ceux-là procurent ainsi au chasseur / héros plus de puissance que les trois animaux herculéens pour qu'il puisse les manipuler à sa guise. L'éloge du chasseur repose d'autant plus sur une configuration légendaire de l'image du héros Bambara. La variation des couleurs de chasse est distribuée dans un triangle chromatique implicite fondé sur trois syntagmes / couleurs des animaux /, couleur de soleil,/ le pelage.

(Invocation du paradis perdu...) $\quad$ Dr. Amgad El-Zarif 
Celui-ci représente pour le chasseur-héros une sorte de déguisement dans la savane. La capacité chromatique afflige le lecteur d'une sorte de cécité temporaire des couleurs intenses au milieu naturel de Ségou, afin que Condé efface de la mémoire du lecteur cette image morne représentée dans le syntagme nominal stéréotypé l'Afrique noire.

Tout compte fait, le chant de louange exalte non seulement la mémoire du chasseur-héros, mais aussi la mère/nature, riche d'animaux sauvages, couleurs vives et secrets innombrables. L'envers du décor naturel et farouche de Ségou se dessine selon la figure ci-dessous:

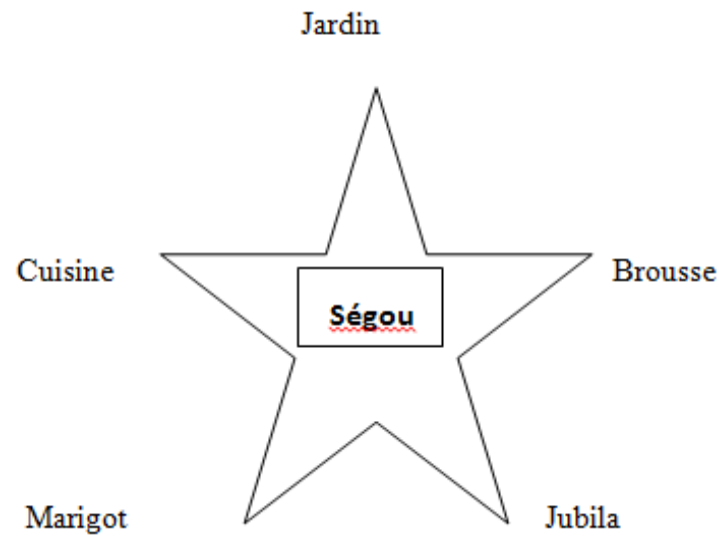

\section{b. Ranimation de la photo bambara}

En tant qu'auteure, Condé paraît comme un électron libre du paysage photographique de Ségou. Dans les énoncés connotés des chants, elle esquisse un hologramme $^{21}$, des pistes d'images abandonnées et des illustrations, formations, montages et diffusions imagées sur les murailles de Ségou. 
Le schéma détendu et itératif du chant correspond à l'état de la convocation silencieuse des photos des ancêtres pour que cette coloration sémantique aide non seulement au transfert du héros épique représenté dans le syntagme métonymique "région d'en bas", qui désigne les lieux de sépulture des ancêtres, mais aussi à la séparation de l'âme au corps, incarné dans la métaphore limpide "son esprit voyagé. " Cette séparation transporterait spirituellement Koumaré aux tombes de la famille royale pour chercher du soutien à restaurer le super-portrait absenté des morts.

Les quatre composants magiques sont précisément sélectionnés pour construire les tubes de coloration à l'esprit du héros, le poème devient ainsi une caméra argentique qui transmet des images photographiques successives des grands-parents dans l'esprit du lecteur, distribuées, colorées selon le tableau ci-dessous:

\begin{tabular}{|c|c|c|c|}
\hline Espèce & Dose & Couleur & Effet / Goût \\
\hline $\begin{array}{c}\text { Plante } \\
\text { (cailcédrat) }\end{array}$ & écorces & grise & amer \\
\hline $\begin{array}{c}\text { Animal } \\
\text { (phacochère) }\end{array}$ & poils & marron & $\begin{array}{c}\text { grande } \\
\text { résistance }\end{array}$ \\
\hline $\begin{array}{c}\text { Animal } \\
\text { (lion) }\end{array}$ & poudre de cour & blanche & courage \\
\hline $\begin{array}{c}\text { Humain } \\
\text { (femme) }\end{array}$ & gouttes de sang & rouge & $\begin{array}{c}\text { procuration des } \\
\text { âmes }\end{array}$ \\
\hline
\end{tabular}

D'autre part, la déglutition volontaire des composantes magiques: écorces, poils poudre de cour, gouttes de sang séquestre le personnage dans un état de 
délire obligatoire et renforce le désir d'invoquer, de dessiner, et de solliciter l'aide des ancêtres. Le vocabulaire des ingrédients de la recette magique relève d'une tradition intellectuelle enfoncée dans les sphères particulières de Ségou véhiculant sur la réincarnation des ancêtres. En plus, le chant échappe aux cadres de réflexion d'une époque révolue pour dépister les conditions des héros bambaras. Ces figures magistrales de la saga humaine de Ségou $^{22}$ mettent en cause la civilisation occidentale.

D'un autre côté, cette photographie vive est mélangée d'un tape-à-l'œil destiné à convoquer le héros comme "encore un enfant" qui contrepointe à l'image vibrante dans "fort de sa chasse". La sublimation du héros dans l'image animale du lion s'est développée dans les deux constructions verbales, Délaissant les biens des hommes et Se repaît de ce qui vit en liberté, illustrant le noyau sémantique de / abstinence/.

La photo parfumée de l'odeur de la mère se sentait dans les termes du chant. Le trait sémantique /maternité/, prévue dans une association sémantique spontanée dans:

$$
\text { Allons mon bébé (bis) }
$$

(SMT, 107)

Le lexème de parenté, bébé, l'impératif permissif aller, l'adjectif possessif mon créent une cajolerie merveilleuse et la quiétude au cœur de l'enfant quand la

(Invocation du paradis perdu...) Dr. Amgad El-Zarif 
mère "l'élevait par trois fois vers l'orient et le couchant." (SMT, 107). Or, il s'agit d'un autre effet sémantique négatif du chant qui laisserait un impact négatif qui montrait les sentiments de Naba en forme enfantine, celle de larmes." Naba avait les larmes aux yeux".

Au demeurant, le chant devient une caméra de chasse de plus en plus précise et perfectionnée, pour identifier le mouvement ou le comportement du serpent sur les feuilles mortes. Le niveau chromatique blafard dans le syntagme nominal métaphorique feuilles mortes dont les couleurs fanées indiquent explicitement le sème / mort/, l'anéantissement et la destruction de la famille Traoré, comme un destin inévitable de la saga.

Le niveau symbolique animal revêt ensuite le lexème symbolique serpent en tête $\mathrm{du}$ chant, une connotation négative directement liée à la mort. De plus, Le chant de camouflage revêt des nuances sémantiques de couleurs lui permettant d'être parfaitement dissimulée dans la nature. Le niveau cinétique est entièrement associé à l'organisation des prédicats dans s'en va, nous as-tu laissés, en t'en allant. Ceux-ci ont acquis le sens de la séparation et de la mort : serpent au début du poème, et $O$ $\mathrm{Kou}$, de trois occurrences à la fin. Le héros sort cruellement de cette forêt labyrinthique. Alors, par le piège photographique chanté, le lecteur attend pour visionner les images capturées, afin de sauvegarder le maximum d'images de Ségou.

(Invocation du paradis perdu...) $\quad$ Dr. Amgad El-Zarif 
Néanmoins, un chant commence par un appel au héros Diémogo avec le prénom bambara Koro, qui est un terme polysémique à trois sens dans le bambara, soit " un arbre fruitier,", soit " une sorte de herbe indigène" , ou soit " un syndrome caractérisé par une anxiété provoquée" de Koro par une virgule au premier vers du chant, est à l'origine d'une intensité de la sensation appropriée à la nouvelle de la mort du frère. Nonobstant, le chant se transforme en leçon de morale quand Tiétigui Banintiéni récite: "Il faut que tu apprennes à marcher seul". Le trait sémantique de la / confraternité/ rappelle vigoureusement le rôle du frère dans les trois lexèmes bien organisés frère, besoin, soutien. L'énoncé interrogatif " Vers qui iras-tu à présent?" un énoncé parémiologique ${ }^{23}$ d'une forme d'obligation impersonnelle il faut que fonctionne comme idéologème.

Quoi qu'il en soit, le trait sémantique / tyrannie / de héros bambara choque le lecteur qui déteste l'éloge dégoûtant du héros, issu de la nature de la structure hiérarchique de Ségou. Le chant s'approche du monologue intérieur d'un bambara, plein d'admiration et d'effroi, quand il se souvient de ses éloges comme suit:

\# Prince, prince, géant de ceux de ton espèce

Ton éteinte donne la mort

Tu joues et tu tues

Tu déchires les cours

(Invocation du paradis perdu...) Dr. Amgad El-Zarif 


\section{La mort qui vient de toi est douce et rapide}

(SMT, 285)

L'occurrence du lexème prince, un appel ambitionné, est un catalyseur pour faire filouter Malobali de l'état de détresse d'esclavage dans lequel il se noie. Les détails se fournissent par réduplication dans: géant de ceux de ton espèce. Le sème espèce suggère la représentation animale, réaffirmé par ton éteinte qui expose la force absolue du héros/animal.

Du reste, le paradoxe sémantique se faufile au cœur du poème chanté dans le troisième vers pour justifier l'obéissance complète et l'acceptation de tous les actes du prince, même contradictoires:

\section{Tu/ joues/ et/ tu/ tues \\ Tu/dé/chires/les/cours}

De plus, les oppositions symétriques concèdent son effet symétrique pour réunir ensemble deux unités lexicales opposées, similaires au chant du rap, afin de transmettre au poème une sorte d'ampleur qui ravive les sensations de l'auditeur et lui fait ressentir la grandeur de la représentation épique du héros glorifié. Par sa structure binaire, l'antithèse scinde l'énoncé du dernier vers en deux:

La mort qui vient de toi // est douce et rapide

(Invocation du paradis perdu...) $\quad$ Dr. Amgad El-Zarif 
À vrai dire, cet aspect antithétique permet de produire, par anticipation à l'auditeur, et à travers le héros lui-même, une certaine vision du monde. L'antithèse permet, dans ces conditions, de mettre l'accent sur son pouvoir absolu. Le paradoxe entre la nature de la mort et le sens des prédicats attributifs douce, rapide crée une valeur plutôt tragique et surprenante. Elle braque la lumière sur une qualité génétique de l'image du prince bambara. Le paradoxe particulier de ce dernier vers d'une longueur double par rapport aux deux vers précédents, associé à un parallélisme ou un chiasme, donne plus de force à l'expression de la violence interne du chant.

Du point de vue sémantique, le chant est doté d'un sens ironique avec la répétition du syntagme nominal injonctif "Pauvre bimi!", alors que l'épithète antéposée pauvre, un quasi morphème, fournit un sens métaphorique du malheur du héros de l'exil de la ville/paradis. La construction symétrique de cette périphrase qualificative Pauvre bimi "apparaît comme le pendant de l'épithète ornementale que l'épopée bambara donne." (Jean Derive, 2005 , 6) Le chant garde son vecteur patrimonial verbal. Il est "le miroir des vertus." Il "restaure le passé, garantit le présent, prévient le futur de l'Africain." (Issiaka, 2017, 52).

De plus, l'utilisation du terme calqué bimi de Bambara, qui signifie "petit", a effectivement été insérée à dessein par Condé pour souligner l'impact psychologique du terme chez le héros même. Au fond, le niveau 
sentimental tendre est passé à la moelle épinière du chant à travers une féminité éclairée représentée dans deux lexèmes très proches mère et femme.

Encore que, comme d'habitude, la mère s'associe à un sentiment du goût et une satiété sémantique à travers un énoncé directif : apporter du lait. L'absence d'un effort et la sensualité/ la volupté d'absorption du lait ne rétablit seulement l'image de la mère, mais il crée encore une atmosphère paradisiaque de la sensualité maternelle du chant.

Ainsi, le lexème femme s'est doté d'un autre niveau sensoriel plus complexe: la satisfaction sexuelle du héros, par la présence d'une autre construction infinitive moudre son grain précédée également par la même préposition pour. Cette construction acquiert de plus en plus une valeur connotative représentée dans l'imagination végétale fertile du chant associée au niveau sexuel chez le héros.

Le terme grain s'emporte, entre autres, d'un niveau sémantique additionnel autre que la nourriture, lié à la force du mâchement associé au passage de l'enfance à la jeunesse. Ainsi, les désirs du héros se métamorphosent envers la mère qui est devenue l'aimée. L'ascendant du milieu agricole de Ségou est déterminé par une isotopie sémantique en cinq lexèmes du climat écologique concernant le chant: vent, pluie, arbre, lait, grain. Ces lexèmes constituent des éléments naturels originels qui produisent l'étonnante image sensuelle de Ségou perdue. 
Dans l'ensemble, le sens du chant collectif du mariage doit procurer une certaine bénédiction par laquelle un chœur joue le rôle d'un religieux, qui bénit le héros. Certes, cette bénédiction fait à ce que ce mariage s'accomplisse heureusement, dans un énoncé initiateur: Que ce mariage soit heureux!".

À première vue, cette supplication ne satisfait ni la vanité, ni la fierté du héros, mais le chœur continue à réjouir les oreilles du héros avec les belles aspirations qui se divisent en deux parties: dans le deuxième vers; le souhait porte sur la progéniture exprimée par la synecdoque, en référence aux enfants issus de ce mariage. Le troisième vers porte un autre souhait plus fort. C'est que le désir et le pouvoir d'amour s'atténuent après avoir des enfants, mais ce vœu est considéré comme un talisman qui protège le couple de la séparation et de la passion de l'amour centré autour le sème $f e u$.

Partant de ce fait, cette spontanéité et la simplicité $\mathrm{du}$ chant porte des connotations profondes et une organisation méticuleuse qui raniment la photo bambara et reflètent la profondeur et l'originalité de son chant.

\section{c. Réexposition des images}

Les Ch.B, une véritable galerie de création photographique. Bien plus met à la portée du lecteur un palier pour s'exprimer. L'espace de Ségou est réservé à ce type particulier d'images accessibles. Si Condé se charge de l'enquête, les images proposent une diversité d'un nouveau style de photographies sociales.

(Invocation du paradis perdu...) $\quad$ Dr. Amgad El-Zarif 
À Ségou, Les Ch.B, une succession ininterrompue d'informations, garantissent la cohésion du corps social. Ils bénéficient "d'une économie de signes dignes d'intérêt. » (Aguessy, 1988, 52) Leur richesse linguistique ne joue pas seulement son rôle d'assurance, mais aussi celui de signification. Ils s'inspirent sans aucun doute des "systèmes expressifs qui sous-tendent les formes symboliques, lesquelles leur servent en somme de médiations sensibles. » (Ladrière, 1977, 101)

Dans Les Ch.B, certains syntagmes nominaux enrichissent d'une matière incontournable la fonction prédicative prévue aux verbes dans les énoncés chantés. A titre d'exemple, la convergence lexicale dans corps à corps réussit de rapprocher les lexèmes afin de dépeindre l'effet fort de l'entrelacement; cette locution adverbiale illustre la mêlée violente, acharnée, entre l'animalité et l'humanité.

Ajoutons que la cohésion physique s'harmonise avec Tamani dans sa résonance profonde, et fait jaillir des termes adjacents aux oreilles des auditeurs dans l'objectif de stimuler l'effet épique dominant sur l'âme, et de créer le sentiment d'enthousiasme et l'interaction avec le héros bambara.

Le complexe forestier infléchit le chemin pictural et colorant du chant à travers l'incarnation de la saga héroïque du chasseur sous une forme animale exagérée. Cette exagération de l'image africaine et exotique est enregistrée dans une succession continue illustrant les

(Invocation du paradis perdu...) $\quad$ Dr. Amgad El-Zarif 
qualités héroïques épiques par le syntagme nominal victorieux de la brousse, lorsque l'énoncé informatif, Tiéfolo porte le lion chassé en travers des épaules, ajoute l'identification corporelle de ce héros. Celui-ci se transforme en lion tout en ayant ses traits physiques. Cette imagerie rend ce qui a été pris indûment de l'étrange "hybridation avec les obsessions personnelles" (López, 419) du héros bambara.

Encore le chant souligne- $\mathrm{t}$ - il que le thème de la métamorphose en animal (Jost, 347, 2005) est ancré dans la littérature orale africaine, même avant la littérature occidentale. En termes de coloration extérieure, Tiéfolo se décrit dans deux énoncés inchoatifs du chant: Tiéfolo est beau. Tiéfolo est brave dont le copule être prête sa fonction prédicative aux deux attributs beau et brave. Ceux-ci accourcissent les traits physiques et moraux du héros. La beauté corporelle se dessine assidûment par la construction nominale prestigieuse jaune au reflet fauve. Cette couleur éblouissante développe l'illusion visuelle adéquate pour concevoir une aura de sacralisation et de séduction autour du personnage.

Il est à constater que le chant incarne la grue couronnée, à travers sa symbolique animale, à la manière du bambara. Ainsi, la réexposition dite périphérique se concrétise différemment dans les quatre composants cidessous:

a) l'énoncé introductif de l'état de sanctification et de révérence "Devant les oiseaux divins générateurs du

(Invocation du paradis perdu...) Dr. Amgad El-Zarif 
langage, tous mirent pied à terre cependant que les griots récitaient." (SMT, 760) prépare à la sainteté du chant adressé à cet oiseau absolument glorifié. Le chant avance sa forme sémantique et lexicale, balançant l'état de l'oiseau unique,

b) les griots ${ }^{24}$ se dirigent à la grue couronnée par un acte déclaratif direct de civilité; Salut. La conduite humaine passe par l'expédition, et aussi la demande de bénédiction de l'oiseau issue d'une certitude mythique d'un pouvoir caché au cœur de cet oiseau qui manipule le destin de Samaké,

c) l'organisation lexicale parole, aspect et voix, répartie successivement dans les troisième, quatrième et cinquième vers ayant la molécule sémantique / humanité/, suggère pleinement les potentiels de force de l'identité humaine de l'oiseau, et affirme la croyance des Bambara que "cet oiseau conscient de ses dons, qu'il a la connaissance de lui-même, et de ce fait il serait à l'origine de la parole de Dieu et de la connaissance que l'homme a de Dieu." (Chevalier et Gheerbrant, 1982, 489)

d) Trois épithètes successives ont décoré l'incroyable puissance vitale, la régénération et la beauté de la grue couronnée:

\begin{tabular}{|l|c|l|}
\hline \multicolumn{1}{|c|}{ épithète } & sa position & \multicolumn{1}{|c|}{ sa valeur sémantique } \\
\hline couronnée & postposée & $\begin{array}{l}\text { cratère inhérent de la } \\
\text { dénomination de l'oiseau }\end{array}$ \\
\hline puissante & postposée & $\begin{array}{l}\text { détermination du pouvoir } \\
\text { magique de l'oiseau }\end{array}$ \\
\hline bel & postposée & $\begin{array}{l}\text { représentation en couleur } \\
\text { étonnante }\end{array}$ \\
\hline
\end{tabular}

(Invocation du paradis perdu...) Dr. Amgad El-Zarif 
Avec la complexité qu'il contient dans toutes les parties de son corps, la grue couronnée a atteint l'équivalent religieux sacré de la tribu.

D'ailleurs, l'image descriptive, signalétique et qualificative de la mort de Mansa, périphrasée touche toute la communauté de Ségou ; elle se formule dans la tournure lexicale décalquée formulaire sur-la-peau-debœuf. Celui-ci s'oppose à l'image grotesque et désopilante abrégée de Pemba. La reproduction du chant de funérailles prend une allure satirique d'un personnage héroïque à travers la mise en place d'une gloire superstitieuse matérialisée dans les trois syntagmes nominaux suivants: "constructeur des choses, choses de l'univers, son pouvoir" distribués régulièrement dans les trois premiers vers du poème.

Cependant, le championnat rocambolesque est vite heurté par l'apparition du Mansa, le vrai héros de Ségou. Ainsi, les femmes annoncent le prétexte de la synthèse précédente, et leurs fausses significations deviennent claires. Cette logique inversée concerne notamment la mort de Demba. Mais pourquoi toute cette ironie et cette félicité dans la mort de ce personnage? La réponse est logique dans l'avant-dernier vers dans l'énoncé assertif emprunté au bambara: "s'assied sur-la-peau-de-boeuf". Cette hardiesse dans l'occupation du siège du roi n'était destinée qu'à la colère des dieux sur cet intrus dans trois images physiques horribles qui détruisent le corps et le cadavre de Demba/Pemba "un flot de sang jaillissant de sa bouche, son visage couvert de pustules, son cadavre

(Invocation du paradis perdu...) Dr. Amgad El-Zarif 
exhalé une terrible puanteur." De ce fait, le chant devient un sermon qui s'adresse à tous ceux qui pensent à s'approcher du Saint-Siège du Roi.

Par le truchement de ses traits sémantiques, le chant découvre son innovation merveilleuse qui perpétue les modes de vie au paradis:

Il a bien raison, le roi, de nous battre.

$$
[\ldots]
$$

Il enveloppe pour nos des femmes à la peau claire

Et les fait entrer ans les chambres nuptiales,

Il achète des noix de Kola pour nous les faire

roquer,

(SMT, 447)

Une sorte de métrage d'animation sensible, les traits paradisiaques du chant se précisent dans trois syntagmes nominaux: La Noix de Kola, La chambre nuptiale, Le destrier. Ces trois structures nominales donnent la vie à des sentiments puissants associés à une augmentation progressive des sensations physiques et morales du Bambara. Nous nous rendons compte que le sème composé la Noix de Kola, étymologiquement emprunté à un dialecte d'Afrique de l'Ouest "le temné", est apprécié grâce à ses vertus stimulantes, ses propriétés stimulantes, antidépressives et aphrodisiaques. 
Malgré son goût amer, la Noix de Kola a une forte teneur en caféine. Ce sème se réfère symboliquement à l'augure favorable dans le folklore des Ch.B. Il se renvoie également à l'amitié partagée, l'entente scellée, la réconciliation dans le paradis.

Quant au syntagme nominal, la chambre nuptiale $^{25}$, il ritualise le mariage très particulier à Ségou portant le symbolisme d'une vie distinctive au sein de la ville. Pour le destrier, un lexème dérivé, dont l'emploi revêt la suprématie militaire de la chevalerie.

Outre qu'il détermine les trois syntagmes nominaux fournis ci-dessous d'une mémoire efficace, Condé plonge l'accession des effets sensoriels et mentaux ainsi que les progrès réalisés dans le chant à travers l'attachement de chaque syntagme à un prédicat très particulier récapitulé selon le tableau ci-après :

\begin{tabular}{|c|c|c|}
\hline le syntagme nominal & le prédicat & L'effet sémantique sensoriel \\
\hline Noix de Kola & faire roquer & $\begin{array}{l}\text { l'accotement l'ethnobotanique à } \\
\text { la culture bambara }\end{array}$ \\
\hline chambres nuptiales & $\begin{array}{l}\text { envelopper pour } \\
\text { fait entrer }\end{array}$ & la virginité des femmes bambara \\
\hline destriers & faire chevaucher & $\begin{array}{l}\text { le dynamisme et la viabilité du } \\
\text { héros bambara. }\end{array}$ \\
\hline
\end{tabular}

Précédé par faire à valeur auxiliaire, les quatre prédicats ont créé le dynamisme interne du texte lyrique, 
le plus nécessaire pour le faire revivre de la viabilité du chant.

L'image mentale se complète par l'extension d'une construction "femmes à la peau claire" se penchant sur les détails du charme physique de la femme bambara. Celle-ci révèle l'image féministe souhaitée dans l'imaginaire du héros. Cette configuration se prolonge à l'esprit du héros quand il se rappelle au chant allégoriquement érotique ci-dessous:

Tes deux seins sont comme deux faons

Jumeaux d'une gazelle

Qui paissent au milieu des lys...

(SMT, 404)

La comparaison extraite du monde animal illustre avec subtilité l'image idéaliste des femmes bambara désirée du héros bambara. Prolongée en deux vers, la comparaison révèle la dégradation illustrée, la douceur du sein de la femme comme signe distinctif au corps nanti de la beauté physique. Les seins moulés comme les plis soyeux des faons Jumeaux d'une gazelle, mais elle a le visage d'une petite fille. L'émergence spatiale à travers la localisation déterminée au milieu des lys... suivie de trois points de suspension ajoute un resplendissement plus glorieusement à la clarté vive des seins. 
Par le biais du syntagme évocateur Plein de ventres se produit une synecdoque référencée au lexème métonymique ventre, se référant aux troupeaux de bétail d'un caractère agronomique de la gloire d'Alfa. Alors que deux lexèmes de parenté, mes femmes et mes enfants, dénotent deux faces pour la même pièce: le premier se réfère à l'état de polygamie comme source de force pour le héros bambara, alors que l'autre confirme l'extension de sa lignée, et par suite la continuation de sa propriété.

Du point de vue spatial, les frontières du Royaume sont déterminées en deux termes: campements et villages, indiquant le contrôle du héros de la plus grande zone contiguë de terre. Ainsi, ces cinq éléments constituent une source de bonheur pour les héros bambaras; Alfa avoue " la paix [le] comble" parce qu'il a des troupeaux (Plein de ventres), femmes, fils, campements et villages. L'excès quantitatif de ces composants est facilement perceptible par l'épithète répétitive et monotone nombreux (ses) régulièrement ajoutée aux_quatre éléments, et l'adverbe Plein de dessine également le tableau magnifique d'accumulation excessive.

La signature du héros sur les composants de son royaume se délimite par des entités lexicales ou grammaticales de possession: à moi, me, mes (bis), j'ai, disposés dans tous les vers du poème pour qu'elles demeurent un cachet royal qui scelle tous ces éléments. Un chant spontané, concentré et simple a pu servir de

(Invocation du paradis perdu...) Dr. Amgad El-Zarif 
preuve et de témoignage irrécusables des éléments perdus du Royaume de Ségou.

Sans surprise, le passé s'émerge graduellement des éloges des griots. Ce poète, porte-parole officiel du héros, identifie/énumère les cinq jouissances sensorielles. La distribution des vers déterminent les priorités pour la construction du Royaume. Le chant psalmodié assimile, par conséquent, une polysémie complexe et variée.

\section{Conclusion}

En guise de conclusion, les Ch.B dans l'immense tissu diégétique historique de SMT ont pu concevoir une invocation audiovisuelle d'un paradis perdu à travers la composante linguistique (vocale et figurative), pour mettre en évidence les formes de vie, les aspects de cette civilisation, la souffrance de ses héros, leurs différents états psychologiques et leur lien fatidique avec cette ville décadente.

Peu à peu, nous avons découvert que Condé devenait cette poétesse capable à revérifier méthodiquement l'héritage oral de Ségou. Cette fois-ci, dans le cadre d'un roman volumineux, (492 pages), qui a averti l'histoire d'une ville sinistrée à la fin du $\underline{\text { XVIII }}^{\text {ème }}$ siècle, et au début du XIX ème, le chant s'est enfilé régulièrement et automatiquement de la part de l'écrivaine comme " un souci majeur de la rénovation de l'écriture et de l'esthétique romanesques africaines." (Ehora, 2013, 29) 
Tous ces éléments froufroutent la subjectivité rythmique des $\mathrm{ChB}$. Ceux-ci manifestent une forte unité orale évocatrice; chaque chant, varié, distribué, bariolé, et même cohérent voire homogène s'attache au fait que Condé démontre une forte tendance à intégrer des matériaux rythmiques ou stylistiques qui contribuent à une recomposition par emboîtement, de la trame romanesque. Cette avalanche du discours oralisé a présenté une particularité à verbaliser les descriptions des modes de vie à Ségou, des héros, des situations, des sentiments, des émotions, etc.

Les patrons rythmiques ont argué le perceptible des images spécifiques du patrimoine populaire et ont suscité un nouvel auditif (impressions auditives) fort et résonnant au moyen des facteurs accompagnant le chant tissés phonétiquement dans SMT. Le rythme, s'attachant au corps des locuteurs (femmes et griots), fléchit l'oralité configurant sa satiété mélodique, attachée à la corrélation d'un phonostylème: monèmes, phonèmes (voyelles et consonnes), accentuation bilatérale ou ternaire, pauses, intensité et intonation.

Le RS a interprété l'espace de Ségou par la restauration auditive des images du pâturage rythmée par la saison, la surprise sonore de l'onomatopée, la parole rituelle et l'anesthésie acoustique. Le RD a développé les enjeux rythmiques animales (la grue couronnée), la technique orale des mouvements, la grille énonciative de la voix féminine, la courbe intonative descendante, le 
rythme ternaire expressif. Le RO a mis en relief des consonnes fricatives du mouvement de corps et de réaction anti-phonémique, l'intonation ascendante de la tension et la pression psychologique, la résonnance buccale de la voyelle, l'harmonie phonologique, l'insistance rythmique du deuil, Le RN a impliqué la conciliation de l'intonation, le pouvoir gnomique et oratoire, la courbe intonative des mouvements mélodiques ascendants, l'interprétation minimale phonétique.

Le scénario du concours phonétique de petits récits intéressants ce dont le lecteur a besoin pour s'orienter vers les murailles particuliers de Ségou: des consonnes [R] , $[\mathrm{b}],[\mathrm{p}],[\mathrm{f}],[\mathrm{k}],\left[\int\right][\mathrm{v}],[\mathrm{s}],[\mathrm{m}]$ des voyelles $[\mathrm{u}],[\varepsilon],[\mathrm{o}]$, des semi-voyelles [wa], a offert à l'épopée une vocalité orale spontanée d'une occurrence régulière en mettant en jeu tout autant les voix que les corps et l'écoute à la maîtrise des acronymes du chant.

Sinon, le lecteur a été invité à la vérification de l'hypothèse de l'étrangeté de l'image la plus diverse et la plus oblique de la nature des Ch.B. Le médium visuel, versé dans les chants, produit des traces de lecture spécifiques par la "décidabilité du rapport" (Louvel, 147, 2020) entre chant et image, toujours ébranlée, et se perpétue entre lire et sentir, d'où la production de ces ondes du visible dans le chant ne s'arrêtent pas d'enfiévrer le lecteur. L'interférence de l'africanité provoquée par le dynamisme inhérent au chant crée ce mouvement de vaet-vient d'un medium de voir de près d'une civilisation 
venant de loin, lorsque la configuration se double du désir du lecteur d'entrer dans la peinture, à la manière de Condé.

La spécificité des traits visuels au sein du chant a somptueusement complété la "photographie" et l'a faite d'un programme de modélisation 3DS d'images abandonnées en trois formes: l'envers du décor de Ségou/ réanimation de la photo bambara / réexposition des images.

Bien qu'elle rappelle les traits élégants de la charge connotative, l'étrangéité des images des Ch.B s'est offerte en mutation perpétuelle, ce qui nous conduit à la perception saisissante, surprenante, voire nostalgique des sensations confuses s'enchaînant à une vitesse vertigineuse, dans un mouvement assidu. Celui-ci a pu briser les détails monotones du texte romanesque, afin de nous dessiller les yeux sur un univers de croyances étranges, comme pour mieux incarner le thème obsédant de la chute, qu'il s'agisse de transition intelligible par le lecteur d'une scène à l'autre des étapes de la décadence de cette civilisation.

Si la réflexion sur la liberté d'expression de l'oralité sans contraintes artificielles reflète la spiritualité origine de la primitivité de Ségou, Condé a ainsi pu archiver les Ch.B digne d'admiration et de contemplation. Les Ch.B ont rendu plus aisé à Condé à conférer à l'histoire un sens issu de cet héritage propice qu'elle avait au centre de sa pensée. 
( $\left.{ }^{1}\right)$ Les Bambaras possèdent cet héritage considérable de chants. Ceuxci sont entremêlés de danses où le bambara est parlé au cercle de Ségou dont l'idiome tient à la fois du bambara et du malinké. (Hippolyte Bazin, 1965)

$\left(^{2}\right)$ La doxa "est héritée de la rhétorique et de la philosophie antiques, elle renvoie à l'ensemble des opinions couramment admises, des croyances largement partagées, des savoirs informels diffusés au sein d'une communauté socio-historique et culturelle donnée." (Provenzano, 2020)

$\left(^{3}\right)$ Schaeffer représente le processus de sa stylistique littéraire par:

- les faits proprement linguistiques (phonologiques, syntaxiques et sémantiques) de niveau infraphrastique et phrastique,

- la conception expressive d'un corpus littéraire d'une représentation spontanée de la création littéraire,

- la fonction expressive de ce corpus met l'accent sur l'analyse des procédés stylistiques.

( $\left.{ }^{4}\right)$ La phonostylistique Pierre Roger Léon (Léon 1993, 6) s'intéresse -à la production oralisée et son effet par rapport au discours, -aux signifiants employés pour rendre une fonction émotionnelle, -aux effets stylistiques désignant des attitudes dans des circonstances particulières.

- à l'intégration au message linguistique ou poétique d'une identification à la culture fondée sur un système d'encodage sonore ou oral.

${ }^{5}$ Une "analogie entre la forme phonique et la forme immédiate ou métaphorique du concept signifié." (Guiraud 1978, 125).

$\left({ }^{6}\right)$ Les termes "idéophone" et "idéophonique", largement utilisés dans la langue bambara Un idéophone vise à rendre compte d'une sensation: une odeur, une couleur, une forme ou un son, voire un mouvement.

$\left(^{7}\right)$ Une image provenant de la réflexion de la lumière, la nuance colorée variant selon l'éclairage.

$\left({ }^{8}\right)$ Petit tambour à deux peaux tendues par des cordelettes sur un corps cylindrique en bois largement entaillé en son milieu et qu'on appelle communément "tambour à aisselles."

$\left({ }^{9}\right)$ http://www.oiseaux-birds.com/index.html

$\left({ }^{10}\right)$ Pour C. Hagège, les idéophones, qui illustrent "le pouvoir évocateur des sons", et sont "un type d'onomatopées", "se servent d'articulations ou de combinaisons phoniques, expressives du fait de 
leur relative rareté, pour mettre en langue des impressions sensorielles ou mentales particulières, liées à certains objets, mouvements ou situations." $(1985,122)$

${ }^{11}$ Kou: la mort, en fon.

$\left(^{r r}\right)$ Fado, un genre musical portugais, constitue de chants populaires au thème mélancolique accompagné d'instruments

$\left({ }^{13}\right)$ Expression qui désigne le Mansa.

$\left({ }^{14}\right)$ Women Grinding Millet Banque d'image et photos - Alamy, alamyimages.fr.

$\left({ }^{15}\right)$ Les griots sont "l'artisan du verbe" [...] ils a longtemps gardé "le monopole du jeu des instruments mélodiques, seuls quelques tambours pouvant être battus par des non-griots." (Zanetti, 201, 161)

$\left({ }^{16}\right)$ Mali Africa Instrument Banque d'image et photos - Alamy alamyimages.fr

$\left({ }^{17}\right)$ Cet instrument de musique donne plus de poids à " sa fonction sociale d'intermédiaire, comme support du message, comme l'assaisonnement indispensable qui donne encore plus de valeur à un chant déjà prestigieux." (Vincent Zanetti, 164)

$\left({ }^{18}\right)$ Mali Africa Instrument Banque d'image et photos - Alamy alamyimages.fr

${ }^{19}$ Pâte e farine de mil, plat très apprécié pour les bambaras

$\left({ }^{20}\right)$ Nom bambara du fleuve Niger.

$\left({ }^{21}\right)$ Cliché photographique transparent ayant enregistré un phénomène de diffraction de la lumière au contact d'un objet à trois dimensions, et qui, illuminé sous un certain angle par un faisceau de lumière, restitue une image en relief de l'objet photographié.

$\left.{ }^{22}\right)$ Pour éterniser des lieux de mémoire, les œuvres de Condé " mettent en évidence les villes africaines [...] Il s'agit [...] de Ségou, [...] de Saint-Louis du Sénégal, etc.[...] elles symbolisent ce port, [...] où les liens étaient définitivement rompus avec la terre natale. " (Kakassa, 2012, 196)

${ }^{23}$ L'idéologème (est) un microsystème sémiotico-idéologique sousjacent à une unité fonctionnelle et significative du discours. Ce système s'organise autour de dominantes sémantiques et d'un ensemble de valeurs qui fluctuent au gré des circonstances historiques. (cf., Cros, 2003 161-181)

$\left({ }^{24}\right)$ Le chant du griot relève son expérience de la hiérarchie sociale du bambara. Sa voix reste la voie de l'argumentation le l'identité 
culturelle, du maintien de la vérité historique de Ségou. Sa parole est "le miroir des vertus." Il "restaure le passé, garantit le présent, prévient le futur de l'Africain." (Issiaka, 2017 : 52)

$\left({ }^{25}\right.$ ) Ce syntagme signifie "le kognosso" par le bambara, fait partie des traditions de mariage de coutume à Ségou avec ses détails: une natte, une moustiquaire blanche, "la Mangnon Magan" (=la vielle dame d'expériences de foyer assiste la jeune mariée pendant la chambre nuptiale), vérification de la virginité de la mariée d'un drap blanc. 


\section{NOTES BIBLIOGRAPHIQUES}

\section{Corpus De l'étude}

- Maryse Condé (1984), Ségou, Les Murailles de terre, Robert Laffont, Paris.

\section{Ouvrages consacrés à la littérature africaine}

- Aguessy, H., (1988), « Tradition orale modèle de culture », in La tradition orale Source de littérature contemporaine en Afrique, N.E.A, ALAIN, Gallimard, Paris.

- Baumgardt, U., et Derive, J., (dir.). (2005) Paroles nomades, écrits d'ethnolinguistique africaine. Paris, Karthala.

- Cisse, M., (2006), Identité créole et écriture métissée dans les romans de Maryse Condé et Simone Schwarz-Bart, thèse de doctorat de lettres et Arts, littérature comparée et francophone, Université Lumière, Lyon 2.

- Condé, M., (2003) Histoire de la femme cannibale. Paris, Mercure de France.

- Dumestre, G., Les idéophones(1998) : le cas du bambara Faits de langues in Les langues d'Afrique subsaharienne.

- Ehora, E., C., (2013), Les «nouveaux habits » de l'oralité chez les romanciers ouest-africains de la seconde génération in Littérature africaine et oralité, Karthala. 
- K. N'Goran, D., (2009), Le champ littéraire africain: Essai pour une théorie, Paris, Le harmattan, coll., critique littéraire.

- Kakassa, A., M., (2012). L'écriture de la mémoire dans le roman africain et antillais contemporain: à propos de Tierno Monénembo et Maryse Condé. Littératures. Université de Lorraine, 2012.

- Ladrière. J., (1977)., Les enjeux de la rationalité, Aubier / Unesco, Paris.

- Poinsot. M., et Treiber. N., (2013) « Entretien avec Maryse Condé in Hommes et migrations, $\mathrm{N}^{\circ} 1301$.

\section{Articles parus en périodiques.}

- Bourlet, M. (2007). L'oralité de la poésie de Bakary Diallo. État d'une recherche en cours, in Études littéraires africaines.

- Buekens, S., (2019), L'écopoétique : une nouvelle approche de la littérature française in Extension du domaine de la littérature,

- DESSONS, G, (2000), Introduction à la poétique. Approche des théories de la littérature, Paris, Nathan.

- $\quad$ Dumestre, G., (1998), Les idéophones : le cas du bambara. In: Faits de langues, $n^{\circ} 11-12$, Octobre 1998.

- Ebongue, A., E., (2013), Quelques aspects lexicaux et syntaxiques de l'oralité et de l'oral dans le texte littéraire d'Afrique francophone, in Synergies Mexique $n^{\circ} 3$

(Invocation du paradis perdu...) Dr. Amgad El-Zarif 
- López, A., P., (2019), Regards sur l'image dans l'écriture de Maryse Condé Thélème (Madr., Internet). 34 (2) .

- Martin, Ph., (2000), L'intonation en parole spontanée", in Française de Linguistique Appliquée, Vol. IV-2.

- Martin-Granel, M., (1993), Roman et oralité, In Cahiers d'études africaines, vol. 33, nº132, 1993. Philippe Boula de Mareuil, Qu'est-ce qu'un (phono) style ? Nouveaux cahiers de linguistique française 3131 (2014)

- Nicolas, M., G., (1993), Roman et oralité.. In: Cahiers d'études africaines, vol. $33, \mathrm{n}^{\circ} 132$.

- Nzessé, L., (2010), Oralité et appropriation du français dans la littérature africaine: le cas de Allah n'est pas obligé d'Ahmadou Kourouma et de La joie de vivre de Patrice Nganang in Synergies Algérie $n^{\circ} 10$.

- Perrin, L. (2012), "L'ethos et le temps fictif de l'oralité à l'écrit ", in Pratiques.

- Pierre Léon, Nouveau regard sur la phonostylistique, in La linguistique, 2009/1 Vol. 45

- Piga, A., (2018), Quelques aspects ethnolinguistiques de l'Afrique sub-saharienne, Aspects linguistiques et sociolinguistiques des français africains in Sapienza Università.

- Rouayrenc, C., $(Y \cdot 10)$ 《Figures et oralité », in Pratiques.

- Schaeffer, J., M., (1997). La stylistique littéraire et son objet. In: littérature, $n^{\circ} 105$ 
- Xavier. G., (2007), Évolution actuelle des littératures africaines. In: Cahiers de l'Association internationale des études françaises, $n^{\circ} 59$.

\section{Ouvrages consacrés à la linguistique}

- Cauvin, J., (1980), Comprendre la Parole traditionnelle, Les classiques africains, Saint-Paul, Paris.

- Chiss, J., L., et Dessons, G., (2000), La force du langage, Honoré Champion, Paris.

- Dessons, G., et Meschonnic, H., (1998), Traité du rythme. Des vers et des proses, Paris, Dunod, 1998.

- Singare, I., A., (2012), L'œuvre poétique de Léopold Sédar Senghor : esthétique de la réception, procès de la création. Linguistique. Université de Cergy Pontoise

- Jean-Marie Schaeffer, J., M., (2015), L'expérience esthétique, Paris, Gallimard, coll. « NRF Essais »,

- Léon, P- R. (1993), Précis de phonostylistique, Paris, Fernand, Nathan.

- Meschonnic, H., (1982), Critique du rythme. Anthropologie historique du langage, Paris, Verdier.

- Meschonnic, H., (1995), Politique du rythme, politique du sujet, Paris, Verdier.

- Zumthor, P., (2008), Oralité, In Intermédialités, Numéro 12, automne 2008.

- Serge, P., et Sénécal M., (1995) « L'interactivité technique, simulacre d'interaction sociale et de démocratie », in : Multimédia et communication à visage humain.

(Invocation du paradis perdu...) $\quad$ Dr. Amgad El-Zarif 
- Ramcy Kabuya,R., (2013) Que reste-t-il de l'oralité dans la littérature africaine contemporaine?, in Revue d'Études Françaises, No 18.

- Arseniev, V., (2007) Les chasseurs Donso du Mali à l'épreuve du temps, in Afrique contemporaine 3-4 $\left(n^{\circ} 223-224\right)$

- Hugo, Z., (1966), La légende des griots malinké. In: Cahiers d'études africaines, vol. 6, n²4,

- Rochmann, M., Ch., (2000), Esclavage et abolitionisme: Mémoires et systèmes de représentation, M-C. Rochmann, éd. Karthala, Paris.

\section{Dictionnaires}

- Dubois, J., Marcellesi, J. B. et Mével, J. P. (1994). Dictionnaire de linguistique et des sciences du langage (Trésors du français), Larousse, Paris.

- $\quad$ Robert, P. et Rey-Derove, J. (2012), Le Petit Robert, Dictionnaire alphabétique et analogique de la langue française, nouvelle édition, Dictionnaires, Le Robert, Paris.

- Bazin, H., (1965), Dictionnaire bambara français, Gregg Presse.

- Jean Chevalier, J., Alain Gheerbrant, A.,(1982) Dictionnaire des symboles, Robert Laffont.

\section{Sitologie}

- Provenzano, F., (2020), « Doxa», dans Anthony Glinoer et Denis Saint-Amand, S., (2020) (dir.), Le lexique socius, URL : http://ressourcessocius.info/index.php/lexique/21-lexique/57-doxa. 
- Derive, J., (2005) La littérature orale de l'œuvre poétique de Senghor: https://halshs.archivesouvertes.fr/halshs-00344027

- Lemaire, M. (1997). Le rythme dans la poésie d'Albert Lozeau. Contribution à l'étude du vers régulier symboliste. Voix et Images, 22 (2), 355-375 https://doi.org/10.7202/201306ar.

- Martin, Ph., (2005), Linguistique de l'oralité : description de la prosodie et analyse instrumentale, Université Paris 7 Denis Diderot, https://www.researchgate.net/publication/278004182.

- Hecquet, V., (2019), Littératures orales africaines, Cahiers d'études africaines [En ligne], 195 | 2009, mis en ligne le 22 septembre 2009, consulté le 30 avril 2019.

- Vincent Zanetti, V., (2011) « Le griot et le pouvoir », Cahiers d'ethnomusicologie [En ligne], 3 |, mis en ligne le15 octobre 2011.

- Jost, M., Deux mythes de métamorphose en animal et leurs interprétations : Lykaon et Kallisto, Madeleine Jost https://doi.org/10.4000/kernos.

- Louvel, L., (2020) Modalités du dialogue entre texte et image. Effets de lecture In : Texte/Image : Images à lire, textes à voir [en ligne]. Rennes : Presses universitaires de Rennes, 2002 (généré le 29 décembre 2020). Disponible sur Internet <http://books.openedition.org/pur/40829>. ISBN : 9782753547407. DOI : https://doi.org/10.4000/books.pur.40829. 
- $\quad$ Île en île, (2013) Maryse Condé : une voix singulière [You tube vidéo]. Disponible sur : https:// www.youtube.com/watch ? v=hAb8RyzR9uo [Dernier accès le 10 septembre 2017].

- Île en île, (2013) Maryse Condé : cinq questions pour Île en l'île [You tube vidéo]. Disponible sur : https://www.youtube.com/watch ? v=41gHapbdkAA [Dernier accès le 15 janvier 2018]

Moura J., M., $(r \cdot 1 r)$, Littératures francophones et théorie postcoloniale Quadrige, PUF, Paris. Résistance à l'hégémonie. 


\section{استدعاء الجنة المفقودة: أهازيج بامبارا \\ في رواية "سيجو، أسوار الأرض"}

ملخص

تشكل اهازيج (أغاني) البومبار اوثيقة لغوية (إيقاعية ودلالية لملحمة

سيجو العظيمة في نهاية القرن الثامن عثر وبداية القرن التاسع عثر) ؛ لقد

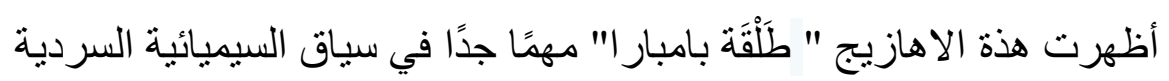
لرواية "سيجو، أسوار الأرض" لقد أصبحت تجسيدًا حيًا وتوضيحًا اقرب الى فيلم رسوم متحركة لتذكير السمعي البصري لمجمل عناصر مملكة سيجو. تعمل هذه الاخيرة الثفوية المهجورة قبل كل شيء كمشّّل عرض لمر احل تألث و انهيار إمبر اطورية البامبار ا. لذلك ليس من المستغرب أن يبدأ الرواية الهائل بأغنية وينتهي أيضًا بأغنية. يقتفى هذا المقال أثر هذه الاهازيج

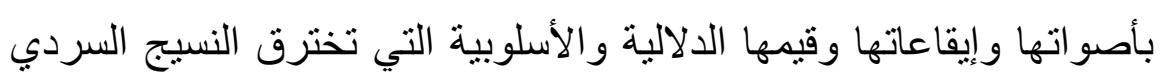
لتخلق إِطْلالات رمزية خاصة. كما ان هذا المقال تححق من ان هذة الاهازيج تشكل عينة بحث شفوية متجانسة أعادت معالجة تحديات (ر هانات) الاستعادة التعويضية لقوة البومابر ا في فضاء غرب إفريقيا.

الكلمات الدالة: شفهي ، أهازيج ، بامبارا ، إيقاع ، الحس ، صورة ، خيال ، إِحْياء 